\title{
Postharvest Treatments on Storage Life of Guava (Psidium guajava L.) in Himalayan Terai Region of West Bengal, India
}

\author{
Piyali Dutta ${ }^{1}$, Nilesh Bhowmick ${ }^{2}$, Surajit Khalko ${ }^{3}$, \\ Arunava Ghosh ${ }^{4}$ and Swapan Kr. Ghosh ${ }^{2}$
}
${ }^{1}$ Department of Fruits and Orchard Management, Bidhan Chandra KrishiViswavidyalaya, Nadia, 741252, West Bengal, India
${ }^{2}$ Department of Pomology and Postharvest Technology, ${ }^{3}$ Department of Plant Pathology, ${ }^{4}$ Department of Agril. Statistics, Uttar Banga Krishi Viswavidyalaya, Pundibari, Cooch Behar, 736165, W.B., India
*Corresponding author

\begin{tabular}{|c|c|}
\hline \multicolumn{2}{|r|}{ A B S T R A C T } \\
\hline & \multirow{6}{*}{$\begin{array}{l}\text { Guava (Psidium guajava L.) is the fifth most important fruit crop of India, has a limited } \\
\text { postharvest shelf life. This study explored the use of some postharvest treatments to extend } \\
\text { the shelf life, post-harvest disease incidence and to assess the physico-chemical changes of } \\
\text { fruits during storage and to study the effect of seasonal variation on quality and storage life } \\
\text { of guava fruits (cv.L-49). Fully mature but green guava fruits were treated with different } \\
\text { edible coating materials, chemicals, powder formulation of bio-control agent, hot water, } \\
\text { and control. On the rainy and winter season, retention percent was found maximum with } \\
\text { paraffin liquid } 10 \% \text {. Physiological loss in weight was minimum with the guar gum } 1 \% \text { in } \\
\text { rainy season, paraffin 5\% and gum acacia } 10 \% \text { in winter season. Regarding other } \\
\text { horticultural traits like total soluble solids, ascorbic acid, total sugar, reducing sugar, non- } \\
\text { reducing sugar content etc. were recorded maximum with gum acacia } 10 \% \text { in rainy season } \\
\text { and winter. From this experiment, it is concluded that winter season guava fruits could be } \\
\text { stored well as compared to rainy season fruits. Edible coating materials showed the better } \\
\text { results in terms of extending the shelf life of guava fruits (cv. L-49) in both rainy and } \\
\text { winter season as well as to maintain the qualities than the other chemicals. }\end{array}$} \\
\hline Keywords & \\
\hline $\begin{array}{l}\text { Edible coatings, } \\
\text { chemicals, bio- } \\
\text { control agent, } \\
\text { guava, Storage Life. }\end{array}$ & \\
\hline Article Info & \\
\hline $\begin{array}{l}\text { Accepted: } \\
\text { 24 February } 2017 \\
\text { Available Online: } \\
10 \text { March } 2017\end{array}$ & \\
\hline & \\
\hline
\end{tabular}

\section{Introduction}

Guava (Psidium guajava L.), belongs to the family Myrtaceae, is the fifth most important fruits of India in terms of production during 2014-15.Guava is one of the commonest fruits liked by poor and the rich people and also known as "The Apple of Tropics" (Bose and Mitra, 2011). Guava is being grown all over the sub-tropical and tropical world due to its high dietary value and good flavor. It is a highly palatable fruit with a rich source of vitamin C (Pal et al., 2004) and it is a climacteric fruits (Akamine and Goo, 1979; Markado-silva et al., 1998); ripen rapidly after the harvest and has a short shelf life. Therefore, guava fruits are required to be managed appropriately in order to get a regulated market supply through postharvest treatments to improve the storage life. Keeping these viewpoints, the study was conducted with objectives to extend the marketable shelf life and to assess the physico-chemical changes of fruits during 
storage and to study the effect of seasonal variation on quality and storage life of guava fruits cv. L-49treated with different edible coating materials (guar gum, gum acacia, chitosan), chemicals (calcium chloride, paraffin liquid), powder formulation of biocontrol agent (Pseudomonas), hot water.

\section{Materials and Methods}

To conduct the experiment, fully mature but green guava fruits (cv. L-49) of two successive seasons (rainy and winter) were collected from an private orchard of Coochbehar district, West Bengal, India during the year 2013 and immediately brought to the laboratory of the Department of Pomology and Postharvest Technology, at Uttar Banga Krishi Viswa vidwalaya, Pundibari, Coochbehar, West Bengal, India for storage after necessary treatments. The fruits after washing in running tap water dried in the shade for few minutes. The fruits were treated with $\mathrm{T}_{1}-\mathrm{CaCl}_{2} 1 \%$ (Dip in aqueous solution of calcium chloride $1 \%$ for five minutes), $\mathrm{T}_{2}-\mathrm{CaCl}_{2} 2 \%$ (Dip in aqueous solution of calcium chloride $2 \%$ for five minutes), $\mathrm{T}_{3}$ - Paraffin liquid 5\% (Dipped for 30 seconds in $5 \%$ aqueous emulsion of liquid paraffin), $\mathrm{T}_{4}$ - Paraffin liquid 10\% (Dipped for 30 seconds in $10 \%$ aqueous emulsion of liquid paraffin), $\mathrm{T}_{5^{-}} \quad$ Pseudomonas fluorescence 1\% (Dipped for 5 minutes in 1\% (Pseudomonas fluorescence) aqueous solution of pseudomonas), $\mathrm{T}_{6^{-}}$Pseudomonas fluorescence 2\% (Dipped for 5 minutes in 2\% (Pseudomonas fluorescence) aqueous solution of pseudomonas), $\mathrm{T}_{7^{-}}$Hot water $\left(45^{\mathrm{O}} \pm 2^{\mathrm{O}} \mathrm{C}\right)$ (Immersed into hot water for 8 minutes at $45^{\mathrm{O}} \mathrm{C} \pm 2^{\mathrm{O}} \mathrm{C}$ in hot water bath chamber and then were hydro cooled rapidly), $\mathrm{T}_{8^{-}}$Hot water $\left(35^{\mathrm{O}} \pm 2^{\mathrm{O}} \mathrm{C}\right.$ ) (Immersed into hot water for 8 minutes at $35^{\circ} \mathrm{C} \pm 2{ }^{\mathrm{O}} \mathrm{C}$ in hot water bath chamber and then were hydro cooled rapidly), $\mathrm{T}_{9^{-}}$Chitosan $0.5 \%$ (To prepare $500 \mathrm{~mL}$ of $0.5 \%(\mathrm{w} / \mathrm{v})$ chitosan solution, accurate weight of $2.5 \mathrm{~g}$ of chitosan were dispersed in $50 \mathrm{~mL}$ of glacial acetic acid), $\mathrm{T}_{10^{-}}$Chitosan $1 \%$ (To prepare $500 \mathrm{~mL}$ of $1 \%(\mathrm{w} / \mathrm{v})$ chitosan solution, accurate weight of $5 \mathrm{~g}$ of chitosan were dispersed in $50 \mathrm{~mL}$ of glacial acetic acid), $\mathrm{T}_{11^{-}}$- Guar gum $1 \%$ (1g guar gum powder was mixed with $100 \mathrm{ml}$ of water for the preparation of $1 \%$ solution), $\mathrm{T}_{12^{-}}$Guar gum 2\% (2g guar gum powder was mixed with $100 \mathrm{ml}$ of water for the preparation of $2 \%$ solutions respectively), $\mathrm{T}_{13^{-}}$Gum acacia $10 \%$ (Dissolve $100 \mathrm{mg}$ of gum acacia powder in $1000 \mathrm{~mL}$ water. The solution was stirred with low heat, $40{ }^{0} \mathrm{C}$ for 30 minutes), $\mathrm{T}_{14^{-}}$ Gum acacia 20\% (Dissolve $200 \mathrm{mg}$ of gum acacia powder in $1000 \mathrm{~mL}$ water. The solution was stirred with low heat at $40{ }^{\circ} \mathrm{C}$ for 30 minutes), $\mathrm{T}_{15^{-}}$Control (Without treatment).

A number of observations were recorded during storage period at an interval of 2 days, i.e., physical parameters of fruits like retention percent of fruits, physiological loss in weight, fruit weight loss $\%=$ (fruit weight at initial period - fruit weight at sampling period)/fruit weight at initial period $\times 100$, diseases incidence percent (percentage of darken fruits due to fungal and mould growth), and bio-chemical parameters of fruits like total soluble solid (TSS) (recorded with the help of handrefractometer, (Mazumdar and Majumder, 2003), total sugar and reducing sugar (AOAC, 1984), ascorbic acid (Rangana, 1977).

\section{Statistical analysis}

Analysis of variance (one way classified data) for each parameter was performed using ProcGlm of Statistical Analysis System (SAS) software (version 9.3). Mean separation for different treatment under different parameter were performed using Least Significant Different (LSD) test $(\mathrm{P} \leq$ 0.05). Data transformation is done by following Gomez and Gomez (Gomez and Gomez, 1983). 


\section{Results and Discussion}

\section{Percentage of retention}

Observation during storage of rainy and winter season guava fruits revealed that the retention percent (Table 1) was decreased in all the treatments as the storage period progressed. In rainy season, on 10 days after treatment, the retention percent was found highest $(80.33 \%)$ with paraffin liquid $10 \%$, followed by paraffin liquid 5\% (79\%), whereas, it was minimum (42\%) in control. However, in winter season, on 12 days after treatment, the retention percent was found highest (86\%) with paraffin liquid $10 \%$, followed by paraffin liquid 5\% (85.67\%) and statistically at par with the other chemicals, whereas, it was minimum $(74.67 \%)$ in control. In winter season, 12 days after treatment, the chemical property of fruits of all treatments was better but marketable quality decreased. Treatment with hot water (Wang et al., 1963) and coating with wax emulsion (Ayranci and Tunc, 2003) also increased the shelf life of mango fruits.

\section{Weight Loss Percentage}

Weight loss percentage increased significantly with the prolongation of the storage period for all treatments. Normally, the weight loss occurs during the fruit storage due to its respiratory process, the transference of humidity and some processes of oxidation (Ayranci and Tunc, 2003). In rainy season on 10 days after treatment, the physiological loss in weight was found minimum $(7.15 \%)$ with guar gum $1 \%$ and statistically at par with paraffin liquid 5\% (7.39\%), whereas, it was maximum (10.49\%) in control. However, on 12 days after treatment, the physiological loss in weight was found lowest (5.60\%) with both paraffin liquid 5\% and gum acacia 10\%and statistically at par with the paraffin liquid $10 \%(5.62 \%)$ and gum acacia 20\% (5.62\%), whereas, it was maximum (8.00\%) in control (Table 2). The reduction in weight loss was probably due to the effects of these coatings as a semi permeable barrier against oxygen, carbon dioxide, moisture and solute movement, thereby reducing respiration, water loss and oxidation reaction rates (Baldwin, 1999).

\section{Disease incidence percent}

It was found that the postharvest disease of guava was mainly due to anthracnose diseases caused by Gloeosporium psidii. The disease incidence percent was studied by visual observation from infected guava fruits. Observation during storage of rainy and winter season guava fruits revealed that the disease incidence percent (Table III) was increased in all the treatments as the storage period progressed.

In rainy season, on 10 days after treatment, the disease incidence percent was found lowest (4\%) with paraffin liquid 10\% which was statistically at par with paraffin liquid 5\% $(5 \%)$, whereas, it was maximum $(26 \%)$ under control. However, in winter season, on 12 days after treatment, the disease incidence percent was found lowest (5\%) with paraffin liquid 10\%, whereas, it was highest (22\%) under control. Passam (Passam, 1982) also reported that 'Doodooth' mango was highly susceptible to anthracnose but when the fruits were treated with hot water $\left(52 \pm 2^{\mathrm{O}} \mathrm{C}\right)$ containing 500-1000 $\mathrm{mg} / \mathrm{l}$ benomyl the incidence of diseases was reduced.

\section{Total soluble solids (TSS)}

TSS content during winter months was higher in general as compared to fruits harvested during rainy season (Table 4). It was increased up to a certain period and there after decreased in all the treatments as the storage period progressed. 
Table.1 Effect of different treatments on fruit retention (percent)

\begin{tabular}{|c|c|c|c|c|c|c|c|c|c|c|c|}
\hline \multirow{3}{*}{ TREATMENTS } & \multicolumn{11}{|c|}{ Days after treatments } \\
\hline & \multicolumn{3}{|c|}{ Rainy season } & & & \multicolumn{4}{|c|}{ Winter season } & \multirow[b]{2}{*}{10} & \multirow[b]{2}{*}{12} \\
\hline & 2 & 4 & 6 & 8 & 10 & 2 & 4 & 6 & 8 & & \\
\hline $\mathrm{T}_{1}-\mathrm{CaCl}_{2} 1 \%$ & $\begin{array}{l}95.00 \\
(77.19) \\
\text { bc }\end{array}$ & $\begin{array}{l}80.66 \\
(63.93) \\
\text { ef }\end{array}$ & $\begin{array}{l}70.66 \\
(57.21) \\
\mathrm{c}\end{array}$ & \begin{tabular}{|l|}
59.67 \\
$(50.57)$ \\
cdef
\end{tabular} & $\begin{array}{l}52.6 \\
(46.5 \\
\mathrm{cd}\end{array}$ & $\begin{array}{l}95.00 \\
(77.19) \\
\text { bc }\end{array}$ & $\begin{array}{l}92.33 \\
(74.07) \\
\text { abcd }\end{array}$ & $\begin{array}{l}90.00 \\
(71.7) \\
a b c\end{array}$ & $\begin{array}{l}87.67 \\
(69.54) \\
a b c\end{array}$ & $\begin{array}{l}85.00 \\
(67.28) \\
\mathrm{a}\end{array}$ & $\begin{array}{l}81.33 \\
(64.5) \\
a b c\end{array}$ \\
\hline $\mathrm{T}_{2^{-}} \mathrm{CaCl}_{2} 2 \%$ & $\begin{array}{l}97.00 \\
(81.85) \\
a b c\end{array}$ & $\begin{array}{l}84.00 \\
(66.51) \\
\text { cde } \\
\end{array}$ & $\begin{array}{l}70.66 \\
(57.22) \\
\mathrm{c} \\
\end{array}$ & \begin{tabular}{|l|}
58.67 \\
$(50)$ \\
def \\
\end{tabular} & $\begin{array}{l}51.67 \\
(45.96) \\
\mathrm{d} \\
\end{array}$ & $\begin{array}{l}97.00 \\
(81.85) \\
\text { ac }\end{array}$ & $\begin{array}{l}93.33 \\
(75.1) \\
\mathrm{abc}\end{array}$ & $\begin{array}{l}91.00 \\
(72.59) \\
\mathrm{abc}\end{array}$ & $\begin{array}{l}88.00 \\
(69.77) \\
\mathrm{abc}\end{array}$ & $\begin{array}{l}85.33 \\
(67.5) \\
\mathrm{a}\end{array}$ & $\begin{array}{l}82.00 \\
(64.94) \\
\mathrm{abc}\end{array}$ \\
\hline $\begin{array}{l}\mathrm{T}_{3^{-}} \text {Paraffin liquid } \\
5 \%\end{array}$ & $\begin{array}{l}100.00 \\
(90) \\
\mathrm{a}\end{array}$ & $\begin{array}{l}94.66 \\
(76.73) \\
\mathrm{a}\end{array}$ & $\begin{array}{l}90.00 \\
(71.56) \\
\mathrm{a}\end{array}$ & \begin{tabular}{|l|}
85.67 \\
$(67.76)$ \\
$\mathrm{a}$ \\
\end{tabular} & $\begin{array}{l}79.00 \\
(62.74) \\
\mathrm{a} \\
\end{array}$ & $\begin{array}{l}100.00 \\
(90) \\
\mathrm{a}\end{array}$ & $\begin{array}{l}97.00 \\
(80.12) \\
\mathrm{a}\end{array}$ & $\begin{array}{l}94.67 \\
(76.66) \\
\mathrm{a}\end{array}$ & $\begin{array}{l}92.33 \\
(73.93) \\
\mathrm{a}\end{array}$ & $\begin{array}{l}89.00 \\
(70.64) \\
\mathrm{a}\end{array}$ & $\begin{array}{l}85.67 \\
(67.76) \\
\mathrm{a} \\
\end{array}$ \\
\hline $\begin{array}{l}\mathrm{T}_{4^{-}} \text {Paraffin liquid } \\
10 \%\end{array}$ & $\begin{array}{l}100.00 \\
(90) \\
\mathrm{a}\end{array}$ & $\begin{array}{l}96.00 \\
(69.06) \\
a\end{array}$ & $\begin{array}{l}93.33 \\
(62) \\
a\end{array}$ & \begin{tabular}{|l|}
87.33 \\
$(56.11)$ \\
$\mathrm{a}$
\end{tabular} & $\begin{array}{l}80.33 \\
(51.74) \\
\mathrm{a}\end{array}$ & $\begin{array}{l}100.00 \\
(90) \\
\mathrm{a}\end{array}$ & $\begin{array}{l}96.67 \\
(79.66) \\
\mathrm{ab}\end{array}$ & $\begin{array}{l}94.33 \\
(76.27) \\
\mathrm{ab}\end{array}$ & $\begin{array}{l}92.00 \\
(73.59) \\
\mathrm{ab}\end{array}$ & $\begin{array}{l}89.00 \\
(70.64) \\
\mathrm{a}\end{array}$ & $\begin{array}{l}86.00 \\
(68.05) \\
\mathrm{a}\end{array}$ \\
\hline $\begin{array}{l}\mathrm{T}_{5^{-}} \quad \text { Pseudor } \\
\text { fluorescence } 1\end{array}$ & $\begin{array}{l}95.33 \\
(80.01) \\
a b c\end{array}$ & $\begin{array}{l}88.33 \\
(70.11) \\
b\end{array}$ & $\begin{array}{l}78.33 \\
(62.47) \\
\mathrm{b} \\
\end{array}$ & \begin{tabular}{|l|}
69.00 \\
$(56.27)$ \\
$\mathrm{b}$ \\
\end{tabular} & $\begin{array}{l}56.67 \\
(48.84) \\
\text { bc } \\
\end{array}$ & $\begin{array}{l}96.00 \\
(78.46) \\
\text { ac } \\
\end{array}$ & $\begin{array}{l}91.33 \\
(72.9) \\
\mathrm{cd}\end{array}$ & $\begin{array}{l}88.67 \\
(70.35) \\
\mathrm{cd}\end{array}$ & $\begin{array}{l}86.00 \\
(68.04) \\
\text { de }\end{array}$ & $\begin{array}{l}83.00 \\
(65.67) \\
\mathrm{ab}\end{array}$ & $\begin{array}{l}80.00 \\
(63.44) \\
\mathrm{abc} \\
\end{array}$ \\
\hline $\begin{array}{l}\mathrm{T}_{6^{-}} \quad \text { Pseudomonas } \\
\text { fluorescence } 2 \%\end{array}$ & $\begin{array}{l}96.00 \\
(78.46) \\
\mathrm{abc}\end{array}$ & $\begin{array}{l}88.66 \\
(70.38) \\
b\end{array}$ & $\begin{array}{l}76.33 \\
(60.89) \\
\text { bc }\end{array}$ & \begin{tabular}{|l|}
65.00 \\
$(53.73)$ \\
$\mathrm{bc}$
\end{tabular} & $\begin{array}{l}54.00 \\
(47.3) \\
\text { bcd }\end{array}$ & $\begin{array}{l}93.33 \\
(77.71) \\
\text { bc }\end{array}$ & $\begin{array}{l}86.33 \\
(68.32) \\
d\end{array}$ & $\begin{array}{l}83.33 \\
(65.95) \\
d\end{array}$ & $\begin{array}{l}81.00 \\
(64.19) \\
d\end{array}$ & $\begin{array}{l}78.00 \\
(62.05) \\
b\end{array}$ & $\begin{array}{l}78.33 \\
(62.46) \\
\mathrm{bc}\end{array}$ \\
\hline $\begin{array}{l}\mathrm{T}_{7^{-}} \quad \text { Hot water } \\
\left(45^{\circ} \pm 2^{\circ} \mathrm{C}\right)\end{array}$ & $\begin{array}{l}95.33 \\
(80.01) \\
a b c\end{array}$ & $\begin{array}{l}85.33 \\
(67.52) \\
\text { bc }\end{array}$ & $\begin{array}{l}70.66 \\
(57.21) \\
\mathrm{c} \\
\end{array}$ & \begin{tabular}{|l|}
57.67 \\
$(49.41)$ \\
ef \\
\end{tabular} & $\begin{array}{l}47.33 \\
(43.47) \\
\mathrm{e}\end{array}$ & $\begin{array}{l}95.33 \\
(79.97) \\
\text { ac } \\
\end{array}$ & & $\begin{array}{l}89.33 \\
(71.14) \\
\text { bcd }\end{array}$ & $\begin{array}{l}86.33 \\
(68.44) \\
\text { de }\end{array}$ & $\begin{array}{l}86.67 \\
(69.24) \\
\mathrm{a} \\
\end{array}$ & $\begin{array}{l}82.33 \\
(65.55) \\
\mathrm{ab} \\
\end{array}$ \\
\hline $\begin{array}{l}\mathrm{T}_{8^{-}} \quad \text { Hot water } \\
\left(35^{\circ} \pm 2^{\circ} \mathrm{C}\right)\end{array}$ & $\begin{array}{l}97.66 \\
(84.89) \\
\mathrm{ab}\end{array}$ & $\begin{array}{l}81.33 \\
(64.46) \\
\text { de }\end{array}$ & $\begin{array}{l}71.66 \\
(57.89) \\
\mathrm{c}\end{array}$ & \begin{tabular}{|l|}
57.67 \\
$(49.41)$ \\
ef
\end{tabular} & $\begin{array}{l}44.67 \\
(41.94) \\
\text { ef }\end{array}$ & $\begin{array}{l}97.67 \\
(84.89) \\
\mathrm{ab}\end{array}$ & & & $\begin{array}{l}88.33 \\
(70.11) \\
\text { abc }\end{array}$ & $\begin{array}{l}85.00 \\
(67.27) \\
\mathrm{a}\end{array}$ & $\begin{array}{l}81.00 \\
(64.19) \\
\mathrm{abc}\end{array}$ \\
\hline $\mathrm{T}_{9^{-}}$ & $\begin{array}{l}91.00 \\
(72.59) \\
\mathrm{c} \\
\end{array}$ & $\begin{array}{l}82.00 \\
(64.97) \\
\text { de }\end{array}$ & $\begin{array}{l}71.66 \\
(57.86) \\
\mathrm{c} \\
\end{array}$ & \begin{tabular}{|l|}
60.00 \\
$(54.1)$ \\
$\mathrm{bc}$ \\
\end{tabular} & $\begin{array}{l}52.33 \\
(46.34) \\
d \\
\end{array}$ & $\begin{array}{l}97.67 \\
(84.89) \\
\mathrm{ab} \\
\end{array}$ & & $\begin{array}{l}91.33 \\
(73.17) \\
\text { abc } \\
\end{array}$ & $\begin{array}{l}89.00 \\
(70.78) \\
\mathrm{abc}\end{array}$ & $\begin{array}{l}86.00 \\
(68.1)\end{array}$ & $\begin{array}{l}82.33 \\
(65.18) \\
\mathrm{ab} \\
\end{array}$ \\
\hline $\mathrm{T}_{1}$ & $\begin{array}{l}93.33 \\
(77.7) \\
b c\end{array}$ & $\begin{array}{l}87.33 \\
(69.21) \\
b c\end{array}$ & $\begin{array}{l}73.66 \\
(59.15) \\
\text { bc }\end{array}$ & \begin{tabular}{|l|}
62.67 \\
$(52.34)$ \\
cde
\end{tabular} & $\begin{array}{l}45.3 . \\
(42.3 \\
\text { ef }\end{array}$ & $\begin{array}{l}95.33 \\
(80.01) \\
\text { ac }\end{array}$ & & & $\begin{array}{l}6.67 \\
68.99) \\
c\end{array}$ & $\begin{array}{l}83.33 \\
(66.26) \\
\mathrm{a}\end{array}$ & $\begin{array}{l}0.67 \\
4.19) \\
c\end{array}$ \\
\hline $\mathrm{T}_{11^{-}}$ & $\begin{array}{l}96.66 \\
(83.85) \\
\text { abc }\end{array}$ & $\begin{array}{l}90.66 \\
(60.89) \\
\text { fg }\end{array}$ & $\begin{array}{l}76.33 \\
(60.89) \\
\text { bc }\end{array}$ & $\begin{array}{l}6 \\
(5 \\
\mathrm{bc} \\
\end{array}$ & $\begin{array}{l}57.67 \\
(49.41) \\
\mathrm{b} \\
\end{array}$ & $\begin{array}{l}96.67 \\
(83.85) \\
\text { ac } \\
\end{array}$ & $\begin{array}{l}93.00 \\
(75.26) \\
a b c\end{array}$ & $\begin{array}{l}90.00 \\
(69.69) \\
\mathrm{cd}\end{array}$ & $\begin{array}{l}87.33 \\
(69.34) \\
\text { abc } \\
\end{array}$ & $\begin{array}{l}84.00 \\
(66.59) \\
a b\end{array}$ & $\begin{array}{l}81.66 \\
(64.81) \\
\text { abc } \\
\end{array}$ \\
\hline $\mathrm{T}_{12-}$ - Guar gum $2 \%$ & $\begin{array}{l}98.66 \\
(86.15) \\
a b\end{array}$ & $\begin{array}{l}93.00 \\
(60.89) \\
\text { fg }\end{array}$ & $\begin{array}{l}76.33 \\
(60.89) \\
\text { bc }\end{array}$ & \begin{tabular}{|l|}
65.66 \\
$(54.13)$ \\
bc
\end{tabular} & $\begin{array}{l}J \\
\text { b } \\
\text { b }\end{array}$ & $\begin{array}{l}98.67 \\
(86.15) \\
a b\end{array}$ & $\begin{array}{l}67 \\
3.23) \\
\end{array}$ & $\begin{array}{l}.00 \\
4.82) \\
c\end{array}$ & $\begin{array}{l}90.00 \\
(71.62) \\
a b c\end{array}$ & $\begin{array}{l}86.67 \\
(68.6) \\
\text { a }\end{array}$ & \begin{tabular}{|l|}
84.00 \\
$(66.44)$ \\
$\mathrm{ab}$ \\
\end{tabular} \\
\hline $\begin{array}{l}\mathrm{T}_{13^{-}} \text {Gum acacia } \\
10 \%\end{array}$ & $\begin{array}{l}97.66 \\
(84.89) \\
\mathrm{ab} \\
\end{array}$ & $\begin{array}{l}92.66 \\
(59.38) \\
\mathrm{g}\end{array}$ & $\begin{array}{l}74.00 \\
(59.38) \\
\text { bc } \\
\end{array}$ & \begin{tabular}{|l|}
63.33 \\
$(52.73)$ \\
bcde \\
\end{tabular} & $\begin{array}{l}53.00 \\
(46.72) \\
\mathrm{cd}\end{array}$ & $\begin{array}{l}95.33 \\
(80.01) \\
\text { ac } \\
\end{array}$ & $\begin{array}{l}92.67 \\
(75.13) \\
a b c\end{array}$ & $\begin{array}{l}90.00 \\
(71.95) \\
a b c\end{array}$ & $\begin{array}{l}88.67 \\
(70.66) \\
\text { abc } \\
\end{array}$ & $\begin{array}{l}85.67 \\
(67.89) \\
\mathrm{a} \\
\end{array}$ & \begin{tabular}{|l|}
82.33 \\
$(65.18)$ \\
$\mathrm{ab}$ \\
\end{tabular} \\
\hline $\begin{array}{l}\mathrm{T}_{14^{-}} \text {Gum acacia } \\
20 \%\end{array}$ & $\begin{array}{l}95.33 \\
(80.01) \\
\text { abc }\end{array}$ & $\begin{array}{l}89.66 \\
(59.38) \\
\mathrm{g}\end{array}$ & $\begin{array}{l}74.00 \\
(59.38) \\
\text { bc }\end{array}$ & \begin{tabular}{|l|}
60.00 \\
$(50.77)$ \\
cdef
\end{tabular} & $\begin{array}{l}51.67 \\
(45.95) \\
d\end{array}$ & $\begin{array}{l}96.00 \\
(78.46) \\
\text { ac }\end{array}$ & $\begin{array}{l}93.33 \\
(75.05) \\
a b c\end{array}$ & $\begin{array}{l}90.67 \\
(72.23) \\
a b c\end{array}$ & $\begin{array}{l}87.67 \\
(69.49) \\
\text { abc }\end{array}$ & $\begin{array}{l}85.00 \\
(67.24) \\
\mathrm{a}\end{array}$ & \begin{tabular}{|l|}
82.33 \\
$(65.18)$ \\
$\mathrm{abc}$ \\
\end{tabular} \\
\hline $\mathrm{T}_{15^{-}}$Control & $\begin{array}{l}96.00 \\
(78.46) \\
a b c\end{array}$ & $\begin{array}{l}87.33 \\
(57.89) \\
\mathrm{g}\end{array}$ & $\begin{array}{l}71.66 \\
(57.89) \\
\mathrm{c}\end{array}$ & \begin{tabular}{|l|}
56.33 \\
$(48.64)$ \\
$\mathrm{f}$ \\
\end{tabular} & $\begin{array}{l}42.00 \\
(40.41) \\
\mathrm{f}\end{array}$ & $\begin{array}{l}91.00 \\
(72.56) \\
\mathrm{c}\end{array}$ & $\begin{array}{l}86.67 \\
(68.62) \\
d\end{array}$ & $\begin{array}{l}83.67 \\
(66.17) \\
d\end{array}$ & $\begin{array}{l}80.67 \\
(63.93) \\
d\end{array}$ & $\begin{array}{l}78.00 \\
(62.03) \\
\mathrm{b}\end{array}$ & \begin{tabular}{|l|}
74.67 \\
$(60)$ \\
$\mathrm{c}$ \\
\end{tabular} \\
\hline $\begin{array}{l}\text { Least Significant } \\
\text { Difference } \\
(\mathrm{P} \leq 0.05)\end{array}$ & 11.765 & 3.430 & 3.790 & 3.640 & 2.37 & 11.77 & 6.16 & 5.42 & 4.73 & 5.01 & 5.17 \\
\hline
\end{tabular}


Table.2 Effect of different treatments on physiological loss in weight (percent)

\begin{tabular}{|c|c|c|c|c|c|c|c|c|c|c|c|}
\hline \multirow{3}{*}{ Treatments } & \multicolumn{11}{|c|}{ Days after treatments } \\
\hline & \multicolumn{5}{|c|}{ Rainy season } & \multicolumn{6}{|c|}{ Winter season } \\
\hline & 2 & 4 & 6 & 8 & 10 & 2 & 4 & 6 & 8 & 10 & 12 \\
\hline $\mathrm{T}_{1}-\mathrm{CaCl}_{2} 1 \%$ & $2.32 \mathrm{ab}$ & $3.22 \mathrm{~d}$ & $4.63 \mathrm{de}$ & $\begin{array}{l}\text { 6.52dcd } \\
e^{-6}\end{array}$ & $8.11 \mathrm{c}$ & $1.61 \mathrm{~b}$ & $2.46 \mathrm{c}$ & $3.37 \mathrm{c}$ & $4.36 \mathrm{c}$ & $5.47 \mathrm{~b}$ & $\begin{array}{l}\text { 6.75cde } \\
\text { f }\end{array}$ \\
\hline $\mathrm{T}_{2}-\mathrm{CaCl}_{2} 2 \%$ & $\begin{array}{l}2.07 \mathrm{bc} \\
\mathrm{d}\end{array}$ & $\begin{array}{l}3.07 \mathrm{~d} \\
\mathrm{e}\end{array}$ & $4.48 \mathrm{e}$ & $\begin{array}{l}\text { 6.26cde } \\
\mathrm{f}\end{array}$ & $8.06 \mathrm{c}$ & $1.60 \mathrm{~b}$ & $2.44 \mathrm{c}$ & $3.35 \mathrm{c}$ & $\begin{array}{l}4.33 \mathrm{~d} \\
\mathrm{c}\end{array}$ & $5.43 \mathrm{~b}$ & $\begin{array}{l}\text { 6.70cde } \\
\mathrm{f}\end{array}$ \\
\hline $\mathrm{T}_{3}$ - Paraffin liquid 5\% & $1.51 \mathrm{e}$ & $\begin{array}{l}3.08 \mathrm{~d} \\
\mathrm{e}\end{array}$ & $4.62 \mathrm{de}$ & $5.88 \mathrm{ef}$ & $7.39 \mathrm{de}$ & $\begin{array}{l}1.31 \mathrm{~b} \\
\mathrm{c}\end{array}$ & $2.39 \mathrm{c}$ & $3.27 \mathrm{c}$ & $\begin{array}{l}4.23 \mathrm{~d} \\
\mathrm{c}\end{array}$ & $5.30 \mathrm{~b}$ & $5.60 \mathrm{~g}$ \\
\hline $\begin{array}{l}\mathrm{T}_{4^{-}} \\
10 \%\end{array}$ & $1.68 \mathrm{dc}$ & $\begin{array}{l}2.85 \mathrm{e} \\
\mathrm{f}\end{array}$ & $4.10 \mathrm{f}$ & $5.97 \mathrm{ef}$ & $\begin{array}{l}7.65 \mathrm{~cd} \\
\mathrm{e}^{-1}\end{array}$ & $1.05 \mathrm{c}$ & $2.40 \mathrm{c}$ & $3.28 \mathrm{c}$ & $\begin{array}{l}4.24 d \\
c\end{array}$ & $5.32 \mathrm{~b}$ & $5.62 \mathrm{~g}$ \\
\hline $\begin{array}{l}\mathrm{T}_{5^{-}} \quad \text { Pseudomonas } \\
\text { fluorescence } 1 \%\end{array}$ & $2.08 \mathrm{bc}$ & $\begin{array}{l}3.40 \mathrm{c} \\
\mathrm{d}\end{array}$ & $5.28 \mathrm{~b}$ & $6.97 \mathrm{bc}$ & $8.99 b$ & $1.59 \mathrm{~b}$ & $2.43 \mathrm{c}$ & $3.33 \mathrm{c}$ & $4.31 \mathrm{~d}$ & $5.40 \mathrm{~b}$ & 6.66def \\
\hline $\begin{array}{l}\mathrm{T}_{6^{-}} \quad \text { Pseudomonas } \\
\text { fluorescence } 2 \%\end{array}$ & $1.66 \mathrm{e}$ & $\begin{array}{l}3.39 \mathrm{c} \\
\mathrm{d}\end{array}$ & $5.27 \mathrm{~b}$ & $6.97 \mathrm{bc}$ & $8.98 b$ & $1.61 \mathrm{~b}$ & $2.46 \mathrm{c}$ & $3.36 \mathrm{c}$ & $4.36 \mathrm{c}$ & $5.47 \mathrm{~b}$ & $\begin{array}{l}6.75 \mathrm{cde} \\
\mathrm{f}\end{array}$ \\
\hline $\begin{array}{l}\mathrm{T}_{7-} \quad \text { Hot } \\
\left(45^{\mathrm{O}} \pm 2^{\mathrm{O}} \mathrm{C}\right)\end{array}$ & $2.22 \mathrm{ab}$ & $\begin{array}{l}3.70 \mathrm{c} \\
\mathrm{b}\end{array}$ & $5.03 \mathrm{bc}$ & $6.98 \mathrm{~b}$ & $9.02 b$ & $1.59 \mathrm{~b}$ & $2.42 \mathrm{c}$ & $3.31 \mathrm{c}$ & $\begin{array}{l}4.28 \mathrm{~d} \\
\mathrm{c}\end{array}$ & $5.37 \mathrm{~b}$ & $6.62 \mathrm{f}$ \\
\hline $\begin{array}{l}\mathrm{T}_{8^{-}} \quad \text { Hot water } \\
\left(35^{\mathrm{O}} \pm 2^{\mathrm{O}} \mathrm{C}\right)\end{array}$ & $2.11 b c$ & $3.89 \mathrm{~b}$ & $\begin{array}{l}4.95 b c \\
d\end{array}$ & $7.11 \mathrm{~b}$ & $9.18 b$ & $1.59 \mathrm{~b}$ & $2.42 \mathrm{c}$ & $3.32 \mathrm{c}$ & $4.29 \mathrm{~d}$ & $5.38 \mathrm{~b}$ & $6.64 \mathrm{ef}$ \\
\hline $\mathrm{T}_{9^{-}}$Chitosan $0.5 \%$ & $2.19 \mathrm{ab}$ & $3.79 b$ & $4.84 \mathrm{~cd}$ & $6.94 \mathrm{bcd}$ & $9.12 b$ & $1.59 \mathrm{~b}$ & $\begin{array}{l}2.69 b \\
c\end{array}$ & $\begin{array}{l}3.61 b \\
\mathrm{c}\end{array}$ & $5.18 b$ & $6.38 \mathrm{a}$ & $\begin{array}{l}\text { 6.81bcd } \\
\mathrm{e}^{-}\end{array}$ \\
\hline $\mathrm{T}_{10^{-}}$Chitosan $1 \%$ & $2.20 \mathrm{ab}$ & $\begin{array}{l}3.35 \mathrm{c} \\
\mathrm{d}\end{array}$ & $\begin{array}{l}4.77 \mathrm{~cd} \\
\mathrm{e}\end{array}$ & $7.01 \mathrm{~b}$ & $8.96 b$ & $1.59 \mathrm{~b}$ & $\begin{array}{l}2.69 b \\
\mathrm{c}\end{array}$ & $\begin{array}{l}3.88 \mathrm{a} \\
\mathrm{b}\end{array}$ & $5.18 \mathrm{~b}$ & $6.38 \mathrm{a}$ & $6.82 \mathrm{bcd}$ \\
\hline $\mathrm{T}_{11^{-}}$Guar gum $1 \%$ & $1.40 \mathrm{e}$ & $2.52 \mathrm{f}$ & $3.75 \mathrm{~g}$ & $5.70 \mathrm{f}$ & $7.15 \mathrm{e}$ & $1.59 \mathrm{~b}$ & $2.43 \mathrm{c}$ & $\begin{array}{l}3.89 \mathrm{a} \\
\mathrm{b}\end{array}$ & $\begin{array}{l}5.20 \mathrm{a} \\
\mathrm{b}\end{array}$ & $6.40 \mathrm{a}$ & $6.84 \mathrm{bc}$ \\
\hline $\mathrm{T}_{12^{-}}$Guar gum $2 \%$ & $\begin{array}{l}1.78 \mathrm{~cd} \\
\mathrm{e}^{-}\end{array}$ & $\begin{array}{l}3.12 \mathrm{~d} \\
\mathrm{e}\end{array}$ & 4.61de & $5.89 \mathrm{ef}$ & $7.85 \mathrm{~cd}$ & $1.60 \mathrm{~b}$ & $\begin{array}{l}3.00 \mathrm{a} \\
\mathrm{b}\end{array}$ & $\begin{array}{l}3.93 \mathrm{a} \\
\mathrm{b}\end{array}$ & $\begin{array}{l}5.27 \mathrm{a} \\
\mathrm{b}\end{array}$ & $6.50 \mathrm{a}$ & $6.95 b$ \\
\hline $\mathrm{T}_{13}-$ Gum acacia $10 \%$ & $2.25 \mathrm{ab}$ & $\begin{array}{l}3.17 d \\
e^{-17}\end{array}$ & $\begin{array}{l}4.76 \mathrm{~cd} \\
\mathrm{e}\end{array}$ & $6.09 \mathrm{ef}$ & $8.15 \mathrm{c}$ & $1.08 \mathrm{c}$ & $2.39 \mathrm{c}$ & $3.29 \mathrm{c}$ & $\begin{array}{l}4.26 \mathrm{~d} \\
\mathrm{c}\end{array}$ & $4.74 \mathrm{c}$ & $5.60 \mathrm{~g}$ \\
\hline $\mathrm{T}_{14^{-}}$Gum acacia $20 \%$ & $2.24 \mathrm{ab}$ & $3.30 \mathrm{~d}$ & $4.89 \mathrm{~cd}$ & $6.24 \mathrm{def}$ & $8.15 \mathrm{c}$ & $1.06 \mathrm{c}$ & $2.39 \mathrm{c}$ & $3.30 \mathrm{c}$ & $\begin{array}{l}4.27 \mathrm{~d} \\
\mathrm{c}\end{array}$ & $5.35 b$ & $5.62 \mathrm{~g}$ \\
\hline $\mathrm{T}_{15^{-}}$Control & $2.54 \mathrm{a}$ & $4.31 \mathrm{a}$ & $6.31 \mathrm{a}$ & $8.18 \mathrm{a}$ & $10.49 \mathrm{a}$ & $2.39 \mathrm{a}$ & $3.27 \mathrm{a}$ & $4.24 \mathrm{a}$ & $5.30 \mathrm{a}$ & $6.54 \mathrm{a}$ & $8.00 \mathrm{a}$ \\
\hline $\begin{array}{l}\text { Least } \quad \text { Significant } \\
\text { Difference }(\mathrm{P} \leq 0.05)\end{array}$ & 0.40 & 0.36 & 0.35 & 0.71 & 0.60 & 0.38 & 0.50 & 0.43 & 0.10 & 0.25 & 0.17 \\
\hline
\end{tabular}


Table.3 Effect of different treatments on disease incidence (percent)

\begin{tabular}{|c|c|c|c|c|c|c|c|c|c|c|c|}
\hline \multirow{3}{*}{ Treatments } & \multicolumn{11}{|c|}{ Days after treatments } \\
\hline & \multicolumn{5}{|c|}{ Rainy season } & \multicolumn{6}{|c|}{ Winter season } \\
\hline & 2 & 4 & 6 & 8 & $\mathbf{1 0}$ & 2 & 4 & 6 & 8 & $\mathbf{1 0}$ & 12 \\
\hline $\mathrm{T}_{1}-\mathrm{CaCl}_{2} 1 \%$ & $\begin{array}{l}1 \\
(3.33) \mathrm{a}\end{array}$ & $\begin{array}{l}6 \\
(14.2) \mathrm{a} \\
\mathrm{b}\end{array}$ & $\begin{array}{l}11 \\
(19.62) a\end{array}$ & $\begin{array}{l}16 \\
(23.56) \\
\mathrm{ab}\end{array}$ & $\begin{array}{l}21(27.2 \\
6) \mathrm{ab}\end{array}$ & $\begin{array}{l}1(3.3 \\
3) \mathrm{a}\end{array}$ & $\begin{array}{l}4(11 . \\
74) a b \\
c d\end{array}$ & $\begin{array}{l}9(17.0 \\
2) \mathrm{a}\end{array}$ & $\begin{array}{l}12(20 . \\
51) \mathrm{ab}\end{array}$ & $\begin{array}{l}16(23 \\
.83) \mathrm{a} \\
\mathrm{b}\end{array}$ & $\begin{array}{l}20(26.5 \\
6) \mathrm{ab}\end{array}$ \\
\hline $\mathrm{T}_{2}-\mathrm{CaCl}_{2} 2 \%$ & $\begin{array}{l}1 \\
(3.33) \\
\mathrm{a}\end{array}$ & $\begin{array}{l}5(12.8) \\
\mathrm{a}\end{array}$ & $\begin{array}{l}10(18.20 \\
) \mathrm{ab}\end{array}$ & $\begin{array}{l}15(22.3 \\
2) \mathrm{ab}\end{array}$ & $\begin{array}{l}20(26.4 \\
5) \mathrm{ab}\end{array}$ & $\begin{array}{l}1(3.3 \\
3) \mathrm{a}\end{array}$ & $\begin{array}{l}4(11 . \\
77) a b \\
\text { cd }\end{array}$ & $\begin{array}{l}7(15.2 \\
6) \mathrm{a}\end{array}$ & $\begin{array}{l}11(18 . \\
86) \mathrm{abc}\end{array}$ & $\begin{array}{l}13(21 \\
.30) \mathrm{a} \\
\mathrm{bc}\end{array}$ & $\begin{array}{l}16(23.4 \\
9) \mathrm{bc}\end{array}$ \\
\hline $\begin{array}{l}\mathrm{T}_{3^{-}} \\
5 \%\end{array}$ & $0(0) \mathrm{a}$ & $0(0) \mathrm{e}$ & $0(0) \mathrm{c}$ & $\begin{array}{l}1(3.33) \\
e^{1}\end{array}$ & $5(12.8) \mathrm{e}$ & $0(0) \mathrm{a}$ & $0(0) \mathrm{f}$ & $0(0) \mathrm{d}$ & $\begin{array}{l}1(3.33) \\
f\end{array}$ & $\begin{array}{l}4(10 . \\
96) \text { ef }\end{array}$ & $\begin{array}{l}7(15.18) \\
\text { fgh }\end{array}$ \\
\hline $\begin{array}{ll}\mathrm{T}_{4^{-}} & \text {Paraffin liquid } \\
10 \% & \end{array}$ & $0(0) \mathrm{a}$ & $0(0) \mathrm{e}$ & $0(0) \mathrm{c}$ & $\begin{array}{l}\mathrm{e}^{1(3.33)} \\
\end{array}$ & $\begin{array}{l}4(11.38) \\
\mathrm{e}\end{array}$ & $0(0) \mathrm{a}$ & $0(0) \mathrm{f}$ & $0(0) \mathrm{d}$ & $\begin{array}{l}1(3.33) \\
\mathrm{f}\end{array}$ & $\begin{array}{l}3(7.6 \\
3) \mathrm{f}\end{array}$ & $\begin{array}{l}5(13.11) \\
\mathrm{h}\end{array}$ \\
\hline $\begin{array}{l}\mathrm{T}_{5^{-}} \quad \text { Pseudomonas } \\
\text { fluorescence } 1 \%\end{array}$ & $0(0) \mathrm{a}$ & $\begin{array}{l}2(4.73) \\
\text { cde }\end{array}$ & $2(4.73) \mathrm{c}$ & $\begin{array}{l}6(14.2) \\
\mathrm{cd}\end{array}$ & $\begin{array}{l}10(18.4 \\
4) \mathrm{d}\end{array}$ & $0(0) \mathrm{a}$ & $0(0) \mathrm{f}$ & $\begin{array}{l}2(6.65) \\
b c\end{array}$ & $\begin{array}{l}4(9.42) \\
\text { ef }\end{array}$ & $\begin{array}{l}7(15 . \\
29) \mathrm{de}\end{array}$ & $\begin{array}{l}10(17.8 \\
2) \text { efg }\end{array}$ \\
\hline $\begin{array}{l}\mathrm{T}_{6^{-}} \quad \text { Pseudomonas } \\
\text { fluorescence } 2 \%\end{array}$ & $0(0) \mathrm{a}$ & $0(0) \mathrm{e}$ & $0(0) \mathrm{c}$ & $\begin{array}{l}4(11.38 \\
) \mathrm{d}\end{array}$ & $\begin{array}{l}10(18.2 \\
0) \mathrm{d}\end{array}$ & $0(0) \mathrm{a}$ & $0(0) \mathrm{f}$ & $0(0) \mathrm{d}$ & $\begin{array}{l}1(3.33) \\
\mathrm{f}\end{array}$ & $\begin{array}{l}4(10 . \\
96) \text { ef }\end{array}$ & $\begin{array}{l}7(14.90) \\
\mathrm{gh}\end{array}$ \\
\hline $\begin{array}{l}\mathrm{T}_{7^{-}} \quad \text { Hot water } \\
\left(45^{\mathrm{O}} \pm 2^{\mathrm{O}} \mathrm{C}\right)\end{array}$ & $0(0) \mathrm{a}$ & $\begin{array}{l}2(6.65) \\
\text { abcd }\end{array}$ & $\begin{array}{l}5(12.78) \\
\mathrm{b}\end{array}$ & $\begin{array}{l}11(19.6 \\
2) \mathrm{bc}\end{array}$ & $\begin{array}{l}21(27.2 \\
6) \mathrm{ab}\end{array}$ & $0(0) \mathrm{a}$ & $\begin{array}{l}2(6.6 \\
5) \\
\text { cdef } \\
\end{array}$ & $\begin{array}{l}5(12.7 \\
8) a b\end{array}$ & $\begin{array}{l}10(18 . \\
42) \\
\text { abcd }\end{array}$ & $\begin{array}{l}15(22 \\
.52) \\
\mathrm{ab}\end{array}$ & $\begin{array}{l}18(24.8 \\
7) \mathrm{abc}\end{array}$ \\
\hline $\begin{array}{l}\mathrm{T}_{8^{-}} \quad \text { Hot } \quad \text { water } \\
\left(35^{\mathrm{O}} \pm 2^{\mathrm{O}} \mathrm{C}\right)\end{array}$ & $\begin{array}{l}1 \\
(3.33) \mathrm{a}\end{array}$ & $\begin{array}{l}6(14.2) \\
a b\end{array}$ & $\begin{array}{l}11(19.62 \\
) \mathrm{a}\end{array}$ & $\begin{array}{l}14(21.9 \\
7) \mathrm{ab}\end{array}$ & $\begin{array}{l}22(27.9 \\
3) \mathrm{ab}\end{array}$ & $\begin{array}{l}1(3.3 \\
3) \mathrm{a}\end{array}$ & $\begin{array}{l}6(14 \\
2) a b\end{array}$ & $\begin{array}{l}10(18 \\
58) \mathrm{a}\end{array}$ & $\begin{array}{l}\text { 13(21. } \\
33) \mathrm{ab}\end{array}$ & $\begin{array}{l}16(23 \\
.80) \mathrm{a} \\
\mathrm{b}\end{array}$ & $\begin{array}{l}19(26.1) \\
\mathrm{abc}\end{array}$ \\
\hline $\mathrm{T}_{9}$ - Chitosan $0.5 \%$ & $\begin{array}{l}2 \\
(6.65) \mathrm{a}\end{array}$ & $\begin{array}{l}5(12.7 \\
8) a b c\end{array}$ & $\begin{array}{l}10(18.44 \\
) a b\end{array}$ & $\begin{array}{l}16(23.5 \\
6) \mathrm{ab}\end{array}$ & $\begin{array}{l}23(28.6 \\
3) \mathrm{ab}\end{array}$ & $\begin{array}{l}1(3.3 \\
3) \mathrm{a}\end{array}$ & $\begin{array}{l}2(4.3 \\
0) \text { ef }\end{array}$ & $\begin{array}{l}2(4.73) \\
\mathrm{cd}\end{array}$ & $\begin{array}{l}5(12.8) \\
\text { cde }\end{array}$ & $\begin{array}{l}8(16 . \\
36) \mathrm{cd}\end{array}$ & $\begin{array}{l}11(19.2 \\
2) \mathrm{de}\end{array}$ \\
\hline $\mathrm{T}_{10^{-}}$Chitosan $1 \%$ & $\begin{array}{l}1 \\
(3.33) \mathrm{a}\end{array}$ & $\begin{array}{l}5(12.7 \\
8) \mathrm{abc}\end{array}$ & $\begin{array}{l}10(18.44 \\
) \mathrm{ab}\end{array}$ & $\begin{array}{l}15(22.7 \\
6) \mathrm{ab}\end{array}$ & $\begin{array}{l}19(25.8 \\
2) \mathrm{ab}\end{array}$ & $0(0) \mathrm{a}$ & $0(0) \mathrm{f}$ & $0(0) \mathrm{d}$ & $\begin{array}{l}4(11.3 \\
8) \mathrm{dc}\end{array}$ & $\begin{array}{l}8(16 . \\
6) \mathrm{cd}\end{array}$ & $\begin{array}{l}\text { 11(18.9 } \\
\text { 1)def }\end{array}$ \\
\hline $\mathrm{T}_{11}$ - Guar gum $1 \%$ & $\begin{array}{l}1(3.33) \\
\mathrm{a}\end{array}$ & $\begin{array}{l}4(9.47) \\
\text { abcd }\end{array}$ & $\begin{array}{l}8(15.38) \\
\mathrm{ab}\end{array}$ & $\begin{array}{l}13(20.9 \\
0) \mathrm{ab}\end{array}$ & $\begin{array}{l}18(24.5 \\
5) \mathrm{bc}\end{array}$ & $\begin{array}{l}1(3.3 \\
3) \mathrm{a}\end{array}$ & $\begin{array}{l}3(7.6 \\
3) \mathrm{bcd} \\
\text { e }\end{array}$ & $\begin{array}{l}6(14.2) \\
\mathrm{a}\end{array}$ & $\begin{array}{l}11(19 . \\
09) \text { abc }\end{array}$ & $\begin{array}{l}14(22 \\
.14) \mathrm{a} \\
\mathrm{b}\end{array}$ & $\begin{array}{l}18(25.0 \\
6) \mathrm{abc}\end{array}$ \\
\hline $\mathrm{T}_{12^{-}}$Guar & $\begin{array}{l}1(3.33) \\
\mathrm{a}\end{array}$ & $\begin{array}{l}2(4.73) \\
\text { cde }\end{array}$ & $5(12.8) \mathrm{b}$ & $\begin{array}{l}10(18.2 \\
0) \mathrm{bc}\end{array}$ & $\begin{array}{l}17(23.8 \\
5) \mathrm{bc}\end{array}$ & $\begin{array}{l}1(3.3 \\
3) \mathrm{a}\end{array}$ & $\begin{array}{l}2(4.7 \\
\text { 3)def }\end{array}$ & $\begin{array}{l}5(12.4 \\
7) \mathrm{ab}\end{array}$ & $\begin{array}{l}10(17 . \\
92) a b c \\
\text { d }\end{array}$ & $\begin{array}{l}12(20 \\
.5) b c \\
\text { d }\end{array}$ & $\begin{array}{l}16(23.5 \\
9) \mathrm{bc}\end{array}$ \\
\hline $\mathrm{T}_{13}$ - Gum acacia $10 \%$ & $0(0) \mathrm{a}$ & $\begin{array}{l}5(12.7 \\
8) \mathrm{abc}\end{array}$ & $\begin{array}{l}9(17.02) \\
\mathrm{ab}\end{array}$ & $\begin{array}{l}13(21.0 \\
7) \mathrm{ab}\end{array}$ & $\begin{array}{l}18(24.8 \\
4) \mathrm{bc}\end{array}$ & $\begin{array}{l}1(3.3 \\
3) \mathrm{a}\end{array}$ & $\begin{array}{l}5(12 . \\
78) a b \\
c\end{array}$ & $\begin{array}{l}9(17.0 \\
2) \mathrm{a}\end{array}$ & $\begin{array}{l}12(20 . \\
23) \mathrm{ab}\end{array}$ & $\begin{array}{l}15(22 \\
.75) \mathrm{a} \\
\mathrm{b}\end{array}$ & $\begin{array}{l}18(24.8 \\
4) a b c\end{array}$ \\
\hline $\mathrm{T}_{14^{-}}$Gum acacia $20 \%$ & $0(0) \mathrm{a}$ & $\begin{array}{l}1(3.33) \\
\text { de }\end{array}$ & $2(4.73) \mathrm{c}$ & $\begin{array}{l}6(14.2) \\
\mathrm{cd}\end{array}$ & $\begin{array}{l}12(20.4 \\
1) \mathrm{cd}\end{array}$ & $0(0) \mathrm{a}$ & $\begin{array}{l}1(3.3 \\
3) \text { ef }\end{array}$ & $\begin{array}{l}2(4.73) \\
\mathrm{cd}\end{array}$ & $\begin{array}{l}6(14.2) \\
\text { bcde }\end{array}$ & $\begin{array}{l}12(19 \\
.89) \mathrm{b} \\
\mathrm{cd}\end{array}$ & $\begin{array}{l}15(22.4 \\
7) \mathrm{cd}\end{array}$ \\
\hline $\mathrm{T}_{15^{-}}$Control & $\begin{array}{l}3 \\
(6.15) \mathrm{a} \\
\end{array}$ & $\begin{array}{l}7 \\
(15.6) \mathrm{a} \\
\end{array}$ & $\begin{array}{l}13 \\
(20.79) \mathrm{a} \\
\end{array}$ & $\begin{array}{l}20(26.5 \\
2) \mathrm{a}\end{array}$ & $\begin{array}{l}6 \\
(30.84) \mathrm{a} \\
\end{array}$ & $\begin{array}{l}2(6.6 \\
5) \mathrm{a} \\
\end{array}$ & $\begin{array}{l}7(14 . \\
93) \mathrm{a}\end{array}$ & $\begin{array}{l}11(19 . \\
05) \mathrm{a}\end{array}$ & $\begin{array}{l}15(23 . \\
04) \mathrm{a}\end{array}$ & $\begin{array}{l}19(26 \\
.1) \mathrm{a} \\
\end{array}$ & $\begin{array}{l}22(28.2) \\
\mathrm{a}\end{array}$ \\
\hline $\begin{array}{l}\text { Least } \quad \text { Significant } \\
\text { Difference }(\mathrm{P} \leq 0.05)\end{array}$ & - & 8.27 & 6.59 & 6.07 & 5.17 & - & 7.21 & 6.64 & 7.15 & 5.34 & 4.00 \\
\hline
\end{tabular}


Int.J.Curr.Microbiol.App.Sci (2017) 6(3): 1831-1842

Table.4 Changes in total soluble solids (Obrix)

\begin{tabular}{|c|c|c|c|c|c|c|c|c|c|c|c|}
\hline \multirow{3}{*}{ Treatments } & \multicolumn{11}{|c|}{ Days after treatments } \\
\hline & \multicolumn{5}{|c|}{ Rainy season } & \multicolumn{6}{|c|}{ Winter season } \\
\hline & 2 & 4 & 6 & 8 & 10 & 2 & 4 & 6 & 8 & 10 & 12 \\
\hline $\mathrm{T}_{1}-\mathrm{CaCl}_{2} 1 \%$ & $\begin{array}{l}6.86 a \\
b c\end{array}$ & 7.30de & $6.43 \mathrm{cdef}$ & $\begin{array}{l}5.85 b c \\
d\end{array}$ & $\begin{array}{l}5.29 \mathrm{~b} \\
\mathrm{c}\end{array}$ & $9.96 \mathrm{cdef}$ & $\begin{array}{l}10.60 \mathrm{c} \\
\mathrm{d}\end{array}$ & $\begin{array}{l}11.26 \mathrm{c} \\
\text { def }\end{array}$ & $\begin{array}{l}11.73 \mathrm{de} \\
\mathrm{fg}\end{array}$ & $\begin{array}{l}10.73 \mathrm{c} \\
\mathrm{de}\end{array}$ & $\begin{array}{l}10.33 \mathrm{c} \\
\mathrm{d}\end{array}$ \\
\hline $\mathrm{T}_{2}-\mathrm{CaCl}_{2} 2 \%$ & $\begin{array}{l}6.77 \mathrm{a} \\
\mathrm{bc}\end{array}$ & $\begin{array}{l}7.37 \mathrm{~cd} \\
\mathrm{e}\end{array}$ & $6.43 \mathrm{cdef}$ & $5.78 \mathrm{~cd}$ & $\begin{array}{l}5.33 b \\
c\end{array}$ & $\begin{array}{l}10.40 \mathrm{ab} \\
\mathrm{c}\end{array}$ & $\begin{array}{l}10.73 a \\
\text { bcd }\end{array}$ & $\begin{array}{l}11.12 \mathrm{~d} \\
\text { ef }\end{array}$ & $\begin{array}{l}11.66 \mathrm{ef} \\
\mathrm{g}\end{array}$ & $10.40 \mathrm{e}$ & $\begin{array}{l}10.00 \\
\text { de }\end{array}$ \\
\hline $\begin{array}{l}\mathrm{T}_{3^{-}} \quad \text { Paraffin } \\
\text { liquid } 5 \%\end{array}$ & $7.19 \mathrm{a}$ & $7.73 \mathrm{ab}$ & $6.91 \mathrm{abc}$ & $6.48 \mathrm{a}$ & $5.86 \mathrm{a}$ & $10.66 \mathrm{ab}$ & $\begin{array}{l}11.27 \mathrm{a} \\
\mathrm{b}\end{array}$ & $\begin{array}{l}11.79 a \\
b\end{array}$ & $\begin{array}{l}12.26 \mathrm{ab} \\
\mathrm{c}\end{array}$ & $11.90 \mathrm{a}$ & $\begin{array}{l}11.13 a \\
b\end{array}$ \\
\hline $\begin{array}{l}\mathrm{T}_{4^{-}} \quad \text { Paraffin } \\
\text { liquid } 10 \%\end{array}$ & $7.16 \mathrm{a}$ & $\begin{array}{l}7.67 \mathrm{ab} \\
\mathrm{c}\end{array}$ & $\begin{array}{l}6.78 \mathrm{abc} \\
\mathrm{d}\end{array}$ & $6.25 \mathrm{ab}$ & $\begin{array}{l}5.63 a \\
b\end{array}$ & $10.66 \mathrm{ab}$ & $\begin{array}{l}11.27 \mathrm{a} \\
\mathrm{b}\end{array}$ & $11.89 \mathrm{a}$ & $12.40 \mathrm{ab}$ & $11.93 \mathrm{a}$ & $\begin{array}{l}11.13 a \\
b\end{array}$ \\
\hline $\begin{array}{l}\mathrm{T}_{5^{-}} \\
\text {Pseudomonas } \\
\text { fluorescence } 1 \\
\%\end{array}$ & $\begin{array}{l}7.01 \mathrm{a} \\
\mathrm{b}\end{array}$ & 7.30de & $6.32 \mathrm{def}$ & $5.97 \mathrm{bc}$ & $5.19 \mathrm{c}$ & $\begin{array}{l}10.33 \mathrm{ab} \\
\mathrm{cd}\end{array}$ & $\begin{array}{l}10.73 a \\
\text { bcd }\end{array}$ & $\begin{array}{l}11.20 \mathrm{c} \\
\text { def }\end{array}$ & 11.60fg & $\begin{array}{l}10.99 \mathrm{c} \\
\mathrm{d}\end{array}$ & $\begin{array}{l}10.06 \\
\text { de }\end{array}$ \\
\hline $\begin{array}{l}\mathrm{T}_{6^{-}} \\
\text {Pseudomonas } \\
\text { fluorescence } \\
2 \%\end{array}$ & $\begin{array}{l}6.94 \mathrm{a} \\
\mathrm{bc}\end{array}$ & $\begin{array}{l}7.37 c d \\
e^{-10}\end{array}$ & $\begin{array}{l}\text { 6.54abc } \\
\text { de }\end{array}$ & $5.93 \mathrm{bc}$ & $\begin{array}{l}5.29 b \\
c\end{array}$ & $9.93 \mathrm{cdef}$ & $\begin{array}{l}10.40 \mathrm{c} \\
\mathrm{d}\end{array}$ & $10.84 \mathrm{f}$ & $\begin{array}{l}11.73 \mathrm{de} \\
\mathrm{fg}\end{array}$ & $\begin{array}{l}11.12 \\
\mathrm{bc}\end{array}$ & $\begin{array}{l}10.73 \\
\mathrm{bc}\end{array}$ \\
\hline $\begin{array}{l}\mathrm{T}_{7^{-}} \text {Hot water } \\
\left(45^{\mathrm{O}} \pm 2^{\mathrm{O}} \mathrm{C}\right)\end{array}$ & $\begin{array}{l}6.77 \mathrm{a} \\
\mathrm{bc}\end{array}$ & $7.16 \mathrm{e}$ & $\begin{array}{l}6.45 \mathrm{bcd} \\
\text { ef }\end{array}$ & $5.74 \mathrm{~cd}$ & $\begin{array}{l}5.29 \mathrm{~b} \\
\mathrm{c}\end{array}$ & $\begin{array}{l}10.06 b c \\
\text { def }\end{array}$ & $\begin{array}{l}11.00 \mathrm{a} \\
\mathrm{bc}\end{array}$ & $\begin{array}{l}11.34 b \\
\text { cdf }\end{array}$ & $11.60 \mathrm{fg}$ & $\begin{array}{l}10.60 \\
\mathrm{de}\end{array}$ & 9.86de \\
\hline $\begin{array}{l}\mathrm{T}_{8^{-}} \text {Hot water } \\
\left(35^{\mathrm{O}} \pm 2^{\mathrm{O}} \mathrm{C}\right)\end{array}$ & $\begin{array}{l}6.54 \mathrm{~b} \\
\mathrm{c}\end{array}$ & $7.06 \mathrm{e}$ & $6.16 \mathrm{ef}$ & $5.68 \mathrm{~cd}$ & $5.19 \mathrm{c}$ & $9.53 \mathrm{f}$ & $10.2 \mathrm{dd}$ & $10.99 \mathrm{ef}$ & $11.46 \mathrm{~g}$ & $\begin{array}{l}10.53 \\
\text { de }\end{array}$ & $9.80 \mathrm{e}$ \\
\hline $\begin{array}{l}\mathrm{T}_{9^{-}} \text {Chitosan } \\
0.5 \%\end{array}$ & $6.46 \mathrm{c}$ & $7.27 \mathrm{de}$ & $\begin{array}{l}\text { 6.46abc } \\
\text { def }\end{array}$ & $\begin{array}{l}5.88 b c \\
d\end{array}$ & $\begin{array}{l}5.42 b \\
c\end{array}$ & $9.80 \mathrm{cdef}$ & $\begin{array}{l}10.53 \mathrm{c} \\
\mathrm{d}\end{array}$ & $\begin{array}{l}11.34 b \\
\text { cde }\end{array}$ & $\begin{array}{l}12.00 \mathrm{bc} \\
\text { def }\end{array}$ & $\begin{array}{l}11.20 \\
\mathrm{bc}\end{array}$ & $\begin{array}{l}10.80 \\
\mathrm{bc}\end{array}$ \\
\hline $\begin{array}{l}\mathrm{T}_{10^{-}} \text {Chitosan } \\
1 \%\end{array}$ & $\begin{array}{l}6.86 a \\
b c\end{array}$ & $7.19 \mathrm{e}$ & $\begin{array}{l}\text { 6.63abc } \\
\text { de }\end{array}$ & $\begin{array}{l}5.88 \mathrm{bc} \\
\mathrm{d}\end{array}$ & $\begin{array}{l}5.36 \mathrm{~b} \\
\mathrm{c}\end{array}$ & $9.67 \mathrm{ef}$ & $\begin{array}{l}10.40 \mathrm{c} \\
\mathrm{d}\end{array}$ & $\begin{array}{l}11.12 \mathrm{~d} \\
\text { ef }\end{array}$ & $\begin{array}{l}11.80 \mathrm{~cd} \\
\text { efg }\end{array}$ & $\begin{array}{l}11.12 \\
\mathrm{bc}\end{array}$ & $\begin{array}{l}10.73 \\
\mathrm{bc}\end{array}$ \\
\hline $\begin{array}{l}\mathrm{T}_{11^{-}} \text {Guar gum } \\
1 \%\end{array}$ & $7.26 \mathrm{a}$ & 7.84ab & $6.96 \mathrm{ab}$ & $6.51 \mathrm{a}$ & $5.93 \mathrm{a}$ & $\begin{array}{l}10.20 \mathrm{ab} \\
\text { cde }\end{array}$ & $\begin{array}{l}10.67 b \\
c d\end{array}$ & $\begin{array}{l}11.44 a \\
\text { bce }\end{array}$ & $\begin{array}{l}12.13 \mathrm{ab} \\
\text { cde }\end{array}$ & $11.79 \mathrm{a}$ & $\begin{array}{l}11.06 \mathrm{a} \\
\mathrm{b}\end{array}$ \\
\hline $\begin{array}{l}\mathrm{T}_{12^{-}} \text {Guar gum } \\
2 \%\end{array}$ & 7.13a & $\begin{array}{l}6.54 \mathrm{bc} \\
\mathrm{d}\end{array}$ & $\begin{array}{l}6.73 a b c \\
d\end{array}$ & $6.00 \mathrm{bc}$ & $\begin{array}{l}5.59 \mathrm{a} \\
\mathrm{b}\end{array}$ & $\begin{array}{l}\begin{array}{l}10.13 b c \\
\text { def }\end{array} \\
\end{array}$ & $\begin{array}{l}10.73 a \\
\text { bcd }\end{array}$ & $\begin{array}{l}11.50 \mathrm{a} \\
\text { bcd }\end{array}$ & $\begin{array}{l}12.20 \mathrm{ab} \\
\mathrm{cd}\end{array}$ & $\begin{array}{l}11.60 \mathrm{a} \\
\mathrm{b}\end{array}$ & $\begin{array}{l}10.93 \\
\mathrm{~b}\end{array}$ \\
\hline $\begin{array}{l}\mathrm{T}_{13^{-}} \text {Gum } \\
\text { acacia } 10 \%\end{array}$ & $7.28 \mathrm{a}$ & 7.91a & $6.91 \mathrm{abc}$ & $6.53 \mathrm{a}$ & $5.95 \mathrm{a}$ & $10.80 \mathrm{a}$ & $11.33 \mathrm{a}$ & $11.90 \mathrm{a}$ & $12.60 \mathrm{a}$ & $12.00 \mathrm{a}$ & $11.26 \mathrm{a}$ \\
\hline $\begin{array}{l}\mathrm{T}_{14^{-}} \text {Gum } \\
\text { acacia } 20 \%\end{array}$ & $7.25 \mathrm{a}$ & $7.90 \mathrm{a}$ & $6.98 \mathrm{a}$ & $6.48 \mathrm{a}$ & $5.86 \mathrm{a}$ & $\begin{array}{l}10.33 \mathrm{ab} \\
\mathrm{cd}\end{array}$ & $\begin{array}{l}11.00 \mathrm{a} \\
\mathrm{bc}\end{array}$ & $\begin{array}{l}11.64 a \\
b c\end{array}$ & $12.33 \mathrm{ab}$ & $11.89 a$ & $\begin{array}{l}11.20 \mathrm{a} \\
\mathrm{b}\end{array}$ \\
\hline $\mathrm{T}_{15^{-}}$Control & $\begin{array}{l}6.74 a \\
\text { bc }\end{array}$ & 7.07e & $6.00 \mathrm{f}$ & $5.51 d$ & $5.16 c$ & $9.73 \mathrm{def}$ & $\begin{array}{l}10.53 \mathrm{c} \\
\mathrm{d}\end{array}$ & $\begin{array}{l}11.20 \mathrm{~d} \\
\text { ef }\end{array}$ & 11.60fg & $\begin{array}{l}10.93 \mathrm{c} \\
\mathrm{d}\end{array}$ & $\begin{array}{l}10.33 \mathrm{c} \\
\mathrm{d}\end{array}$ \\
\hline $\begin{array}{l}\text { Least } \\
\text { Significant } \\
\text { Difference } \\
(\mathrm{P} \leq 0.05) \\
\end{array}$ & 0.55 & 0.34 & 0.52 & 0.40 & 0.36 & 0.67 & 0.66 & 0.50 & 0.53 & 0.49 & 0.51 \\
\hline
\end{tabular}

**Means with the same letter are not significantly different 
Int.J.Curr.Microbiol.App.Sci (2017) 6(3): 1831-1842

Table.5 Changes in total sugar content (mg)

\begin{tabular}{|c|c|c|c|c|c|c|c|c|c|c|c|}
\hline \multirow{3}{*}{ Treatments } & \multicolumn{11}{|c|}{ Days after treatments } \\
\hline & \multicolumn{5}{|c|}{ Rainy season } & \multicolumn{6}{|c|}{ Winter season } \\
\hline & 2 & 4 & 6 & 8 & 10 & 2 & 4 & 6 & 8 & 10 & 12 \\
\hline $\mathrm{T}_{1}-\mathrm{CaCl}_{2} 1 \%$ & $\begin{array}{l}4.33 \\
\mathrm{~cd}\end{array}$ & $\begin{array}{l}5.11 \\
\mathrm{bc}\end{array}$ & $\begin{array}{l}4.27 \\
d\end{array}$ & 3.76def & $\begin{array}{l}3.29 \mathrm{~d} \\
\mathrm{e}^{-}\end{array}$ & $6.11 \mathrm{f}$ & $6.56 \mathrm{~h}$ & $6.88 \mathrm{f}$ & $\begin{array}{l}7.17 \\
b c\end{array}$ & $6.49 \mathrm{e}$ & $\begin{array}{l}6.04 \\
\mathrm{~g}\end{array}$ \\
\hline $\mathrm{T}_{2}-\mathrm{CaCl}_{2} 2 \%$ & $\begin{array}{l}4.27 \\
\text { de }\end{array}$ & $\begin{array}{l}5.17 \\
\mathrm{~b}\end{array}$ & $\begin{array}{l}4.30 \\
\mathrm{~cd}\end{array}$ & $3.67 \mathrm{ef}$ & $\begin{array}{l}3.33 \mathrm{~d} \\
\mathrm{e}^{-1}\end{array}$ & $\begin{array}{l}6.67 b c \\
d\end{array}$ & $\begin{array}{l}6.94 \mathrm{~b} \\
\text { cde }\end{array}$ & $\begin{array}{l}7.02 \mathrm{~d} \\
\mathrm{e}^{-1}\end{array}$ & $\begin{array}{l}7.17 \\
\mathrm{bc}\end{array}$ & $\begin{array}{l}6.69 c \\
\text { de }\end{array}$ & $\begin{array}{l}6.29 \\
\text { de }\end{array}$ \\
\hline $\begin{array}{l}\mathrm{T}_{3} \text {-Paraffin liquid } \\
5 \%\end{array}$ & $\begin{array}{l}4.72 \\
\mathrm{a}\end{array}$ & \begin{tabular}{|l|}
5.51 \\
$\mathrm{a}$
\end{tabular} & $\begin{array}{l}4.77 \\
\mathrm{a}\end{array}$ & $4.26 a b$ & $3.89 \mathrm{a}$ & $\begin{array}{l}6.75 \mathrm{ab} \\
\mathrm{c}\end{array}$ & $\begin{array}{l}6.98 \mathrm{a} \\
\mathrm{bc}\end{array}$ & $\begin{array}{l}7.17 \mathrm{a} \\
\mathrm{b}\end{array}$ & $\begin{array}{l}7.27 \\
\mathrm{a}\end{array}$ & $\begin{array}{l}6.95 a \\
b\end{array}$ & $\begin{array}{l}6.60 \\
\mathrm{ab}\end{array}$ \\
\hline $\begin{array}{l}\mathrm{T}_{4} \text { - Paraffin liquid } \\
10 \%\end{array}$ & \begin{tabular}{|l}
4.66 \\
$\mathrm{ab}$
\end{tabular} & \begin{tabular}{|l|}
5.53 \\
$\mathrm{a}$ \\
\end{tabular} & $\begin{array}{l}4.56 \\
\mathrm{~b}\end{array}$ & $\begin{array}{l}4.19 a b c \\
d\end{array}$ & $\begin{array}{l}3.69 \mathrm{a} \\
\mathrm{b}\end{array}$ & $6.92 \mathrm{a}$ & $7.07 \mathrm{a}$ & $7.23 \mathrm{a}$ & $\begin{array}{l}7.29 \\
\mathrm{a} \\
\end{array}$ & $\begin{array}{l}6.92 a \\
b \\
\end{array}$ & $\begin{array}{l}6.62 \\
\mathrm{a}\end{array}$ \\
\hline $\begin{array}{l}\mathrm{T}_{5^{-}} \text {Pseudomonas } \\
\text { fluorescence } 1 \%\end{array}$ & $\begin{array}{l}4.49 \\
\mathrm{bc}\end{array}$ & $\begin{array}{l}5.05 \\
\mathrm{bc}\end{array}$ & $\begin{array}{l}4.23 \\
\mathrm{~d}\end{array}$ & $\begin{array}{l}3.83 \mathrm{bcd} \\
\text { ef }\end{array}$ & $\begin{array}{l}3.19 \mathrm{~d} \\
\mathrm{e}\end{array}$ & $6.83 \mathrm{ab}$ & $\begin{array}{l}6.91 b \\
\text { cde }\end{array}$ & $\begin{array}{l}7.08 \mathrm{c} \\
\mathrm{de}\end{array}$ & $\begin{array}{l}7.18 \\
b c\end{array}$ & $\begin{array}{l}6.62 c \\
\text { de }\end{array}$ & $\begin{array}{l}6.27 \\
\mathrm{e}^{6}\end{array}$ \\
\hline $\begin{array}{l}\mathrm{T}_{6}-\text { Pseudomonas } \\
\text { fluorescence } 2 \%\end{array}$ & $\begin{array}{l}4.38 \\
\mathrm{~cd}\end{array}$ & $\begin{array}{l}5.18 \\
\mathrm{~b}\end{array}$ & $\begin{array}{l}4.35 \\
\text { cd }\end{array}$ & $\begin{array}{l}\text { 3.81cde } \\
\text { f }\end{array}$ & $\begin{array}{l}3.29 \mathrm{~d} \\
\mathrm{e}^{-1}\end{array}$ & $6.52 \mathrm{~d}$ & $\begin{array}{l}6.84 \mathrm{~d} \\
\text { ef }\end{array}$ & $7.00 \mathrm{e}$ & $\begin{array}{l}7.15 \\
\text { cd }\end{array}$ & $\begin{array}{l}6.63 \mathrm{c} \\
\text { de }\end{array}$ & $\begin{array}{l}6.26 \\
e^{6}\end{array}$ \\
\hline $\begin{array}{l}\mathrm{T}_{7^{-}} \quad \text { Hot water } \\
\left(45^{\mathrm{O}_{ \pm}}+2^{\mathrm{O}} \mathrm{c}\right)\end{array}$ & $\begin{array}{l}4.10 \\
\mathrm{e}\end{array}$ & $\begin{array}{l}5.07 \\
\mathrm{bc}\end{array}$ & $\begin{array}{l}4.25 \\
d\end{array}$ & ef & $\begin{array}{l}3.30 \mathrm{~d} \\
\mathrm{e}^{-}\end{array}$ & $6.59 \mathrm{~cd}$ & $6.72 \mathrm{~g}$ & $\begin{array}{l}7.07 d \\
e^{-3}\end{array}$ & $\begin{array}{l}7.25 \\
\mathrm{ab}\end{array}$ & $\begin{array}{l}6.76 \mathrm{~b} \\
\mathrm{~cd}\end{array}$ & $\begin{array}{l}6.41 \\
\mathrm{~cd}\end{array}$ \\
\hline $\begin{array}{l}\mathrm{T}_{8^{-}} \quad \text { Hot } \\
\left(35^{\mathrm{O}_{ \pm}} 2^{\mathrm{O}} \mathrm{c}\right)\end{array}$ & $\begin{array}{l}4.33 \\
\mathrm{~cd}\end{array}$ & $\begin{array}{l}5.11 \\
\mathrm{bc}\end{array}$ & $\begin{array}{l}4.31 \\
\mathrm{~cd}\end{array}$ & $\begin{array}{l}\text { 3.86abc } \\
\text { def }\end{array}$ & $3.16 \mathrm{e}$ & $6.28 \mathrm{ef}$ & $6.57 \mathrm{~h}$ & $6.89 \mathrm{f}$ & $\begin{array}{l}7.08 \\
d\end{array}$ & $\begin{array}{l}6.66 \mathrm{c} \\
\text { de }\end{array}$ & $\begin{array}{l}6.21 \\
\text { ef }\end{array}$ \\
\hline $\begin{array}{ll}\mathrm{T}_{9^{-}} & \text {Chitosan } \\
0.5 \% & \end{array}$ & $\begin{array}{l}4.33 \\
\mathrm{~cd}\end{array}$ & $\begin{array}{l}5.12 \\
\mathrm{bc}\end{array}$ & $\begin{array}{l}4.44 \\
\mathrm{bc}\end{array}$ & 3.75def & $\begin{array}{l}3.43 \mathrm{c} \\
\mathrm{d}\end{array}$ & $\begin{array}{l}6.60 \mathrm{bc} \\
\mathrm{d}\end{array}$ & $\begin{array}{l}6.94 \mathrm{~b} \\
\mathrm{~cd}\end{array}$ & $\begin{array}{l}7.05 \mathrm{~d} \\
\mathrm{e}^{-l^{2}}\end{array}$ & $\begin{array}{l}7.14 \\
\text { cd }\end{array}$ & $\begin{array}{l}6.83 a \\
b c\end{array}$ & $\begin{array}{l}6.46 \\
\mathrm{c}\end{array}$ \\
\hline $\mathrm{T}_{10^{-}}$Chitosan $1 \%$ & $\begin{array}{l}4.40 \\
\mathrm{~cd}\end{array}$ & $\begin{array}{l}5.11 \\
\mathrm{bc}\end{array}$ & $\begin{array}{l}4.50 \\
\mathrm{~b}\end{array}$ & 3.74def & $\begin{array}{l}3.35 c \\
\text { de }\end{array}$ & $\begin{array}{l}6.50 \mathrm{bc} \\
\mathrm{de}\end{array}$ & $\begin{array}{l}6.83 e \\
\text { fg }\end{array}$ & $\begin{array}{l}7.07 d \\
e^{-1}\end{array}$ & $\begin{array}{l}7.14 \\
\mathrm{~cd}\end{array}$ & $\begin{array}{l}6.74 \mathrm{~b} \\
\mathrm{~cd}\end{array}$ & $\begin{array}{l}6.48 \\
\mathrm{bc}\end{array}$ \\
\hline $\begin{array}{lll}\mathrm{T}_{11^{-}} & \text {Guar } \\
1 \% & \text { gum }\end{array}$ & $\begin{array}{l}4.78 \\
\mathrm{a}\end{array}$ & \begin{tabular}{|l|}
5.61 \\
$\mathrm{a}$
\end{tabular} & $\begin{array}{l}4.80 \\
\mathrm{a}\end{array}$ & 4.30ab & $3.93 \mathrm{a}$ & $\begin{array}{l}6.71 \mathrm{ab} \\
\mathrm{cd}\end{array}$ & $\begin{array}{l}6.79 f \\
g\end{array}$ & $\begin{array}{l}7.07 \mathrm{~d} \\
\mathrm{e}^{-l^{2}}\end{array}$ & $\begin{array}{l}7.11 \\
\mathrm{~cd}\end{array}$ & $\begin{array}{l}6.82 \mathrm{a} \\
\mathrm{bc}\end{array}$ & $\begin{array}{l}6.33 \\
\text { de }\end{array}$ \\
\hline 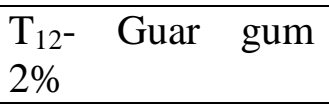 & $\begin{array}{l}4.65 \\
\mathrm{ab}\end{array}$ & $\begin{array}{l}5.49 \\
\mathrm{a}\end{array}$ & $\begin{array}{l}4.58 \\
\mathrm{~b}\end{array}$ & $\begin{array}{l}\text { 4.07abc } \\
\text { de }\end{array}$ & $\begin{array}{l}3.59 \mathrm{~b} \\
\mathrm{c}\end{array}$ & $6.54 \mathrm{~cd}$ & $\begin{array}{l}6.87 \mathrm{c} \\
\text { def }\end{array}$ & $\begin{array}{l}7.03 \mathrm{~d} \\
\mathrm{e}\end{array}$ & $\begin{array}{l}7.12 \\
\mathrm{~cd}\end{array}$ & $\begin{array}{l}6.65 c \\
\text { de }\end{array}$ & $\begin{array}{l}6.40 \\
\mathrm{~cd}\end{array}$ \\
\hline $\begin{array}{l}\mathrm{T}_{13^{-}} \text {Gum acacia } \\
10 \%\end{array}$ & $\begin{array}{l}4.78 \\
\mathrm{a}\end{array}$ & $\begin{array}{l}5.60 \\
\mathrm{a}\end{array}$ & $\begin{array}{l}4.81 \\
\mathrm{a}\end{array}$ & $4.30 \mathrm{a}$ & $3.93 \mathrm{a}$ & $6.93 \mathrm{a}$ & $\begin{array}{l}7.01 \mathrm{a} \\
\mathrm{b}\end{array}$ & $\begin{array}{l}7.17 \mathrm{a} \\
\mathrm{bc}\end{array}$ & $\begin{array}{l}7.28 \\
\mathrm{a}\end{array}$ & $6.98 \mathrm{a}$ & $\begin{array}{l}6.64 \\
\mathrm{a}\end{array}$ \\
\hline $\begin{array}{l}\mathrm{T}_{14-} \text { Gum acacia } \\
20 \%\end{array}$ & $\begin{array}{l}4.70 \\
\mathrm{a}\end{array}$ & $\begin{array}{l}5.61 \\
\mathrm{a}\end{array}$ & $\begin{array}{l}4.80 \\
\mathrm{a}\end{array}$ & $4.25 \mathrm{abc}$ & $3.89 \mathrm{a}$ & $\begin{array}{l}6.63 b c \\
d\end{array}$ & $\begin{array}{l}6.94 \mathrm{~b} \\
\mathrm{~cd}\end{array}$ & $\begin{array}{l}7.10 b \\
\mathrm{~cd}\end{array}$ & $\begin{array}{l}7.24 \\
\mathrm{ab}\end{array}$ & $6.99 \mathrm{a}$ & $\begin{array}{l}6.66 \\
\mathrm{a}\end{array}$ \\
\hline Control & $\begin{array}{l}4.25 \\
\text { de }\end{array}$ & $\begin{array}{l}5.00 \\
\mathrm{c}\end{array}$ & $\begin{array}{l}4.26 \\
d\end{array}$ & $3.60 \mathrm{f}$ & $3.13 \mathrm{e}$ & $6.54 \mathrm{~cd}$ & $\begin{array}{l}6.89 \mathrm{c} \\
\text { def }\end{array}$ & $\begin{array}{l}7.01 \mathrm{~d} \\
\mathrm{e}^{-}\end{array}$ & $\begin{array}{l}7.12 \\
\mathrm{~cd}\end{array}$ & $\begin{array}{l}6.59 d \\
e^{-6.1}\end{array}$ & $\begin{array}{l}6.13 f \\
g\end{array}$ \\
\hline $\begin{array}{l}\text { Least Significant } \\
\text { Difference } \\
(\mathrm{P} \leq 0.05)\end{array}$ & 0.18 & 0.15 & 0.14 & 0.45 & 0.25 & 0.23 & 0.11 & 0.09 & 0.07 & 0.22 & 0.12 \\
\hline
\end{tabular}

**Means with the same letter are not significantly different 
Int.J.Curr.Microbiol.App.Sci (2017) 6(3): 1831-1842

Table.6 Changes in reducing sugar content (mg)

\begin{tabular}{|c|c|c|c|c|c|c|c|c|c|c|c|}
\hline \multirow{3}{*}{ Treatments } & \multicolumn{11}{|c|}{ Days after treatments } \\
\hline & \multicolumn{5}{|c|}{ Rainy season } & \multicolumn{6}{|c|}{ Winter season } \\
\hline & 2 & 4 & 6 & 8 & 10 & 2 & 4 & 6 & 8 & 10 & 12 \\
\hline $\mathrm{T}_{1}-\mathrm{CaCl}_{2} 1 \%$ & $\begin{array}{l}2.30 \mathrm{c} \\
\text { def }\end{array}$ & $\begin{array}{l}3.03 \mathrm{c} \\
\mathrm{e}\end{array}$ & $\begin{array}{l}2.31 \\
\mathrm{~d}\end{array}$ & \begin{tabular}{|l}
2.04 \\
$\mathrm{a}$
\end{tabular} & $\begin{array}{l}1.70 \mathrm{~d} \\
\text { ef }\end{array}$ & $\begin{array}{l}3.15 \mathrm{~d} \\
\text { ef }\end{array}$ & $3.27 \mathrm{ef}$ & $\begin{array}{l}3.3 \\
5 \mathrm{a}\end{array}$ & $\begin{array}{l}3.21 \\
\mathrm{e}\end{array}$ & $\begin{array}{l}3.07 \\
d\end{array}$ & $3.01 d$ \\
\hline $\mathrm{T}_{2}-\mathrm{CaCl}_{2} 2 \%$ & $\begin{array}{l}2.20 \mathrm{~d} \\
\text { efg }\end{array}$ & $\begin{array}{l}3.10 c \\
\text { de }\end{array}$ & $\begin{array}{l}2.37 \\
\mathrm{~cd}\end{array}$ & $\begin{array}{l}2.03 \\
\mathrm{a}\end{array}$ & $\begin{array}{l}1.75 c \\
d e\end{array}$ & $\begin{array}{l}3.16 c \\
\text { def }\end{array}$ & $3.26 \mathrm{ef}$ & $\begin{array}{l}3.3 \\
3 b\end{array}$ & $\begin{array}{l}3.20 \\
\mathrm{e}\end{array}$ & $\begin{array}{l}3.11 \\
\mathrm{~cd}\end{array}$ & $\begin{array}{l}3.02 \mathrm{c} \\
\mathrm{d}\end{array}$ \\
\hline $\begin{array}{l}\mathrm{T}_{3} \text {-Paraffin liquid } \\
5 \%\end{array}$ & $2.57 \mathrm{a}$ & $\begin{array}{l}3.30 \mathrm{a} \\
\mathrm{b}\end{array}$ & $\begin{array}{l}2.70 \\
\mathrm{ab}\end{array}$ & $\begin{array}{l}2.36 \\
\mathrm{a}\end{array}$ & $\begin{array}{l}1.96 \mathrm{a} \\
\mathrm{b}\end{array}$ & $\begin{array}{l}3.23 \mathrm{a} \\
\mathrm{b}\end{array}$ & $\begin{array}{l}3.33 a b \\
c\end{array}$ & $\begin{array}{l}3.4 \\
1 \mathrm{a}\end{array}$ & $\begin{array}{l}3.33 \\
b\end{array}$ & $\begin{array}{l}3.26 \\
\mathrm{a}\end{array}$ & $3.18 \mathrm{a}$ \\
\hline $\begin{array}{l}\mathrm{T}_{4} \text { - Paraffin liquid } \\
10 \%\end{array}$ & $\begin{array}{l}2.53 \mathrm{a} \\
\mathrm{b}\end{array}$ & $\begin{array}{l}3.30 \mathrm{a} \\
\mathrm{b}\end{array}$ & $\begin{array}{l}2.53 \\
\mathrm{bc}\end{array}$ & $\begin{array}{l}2.32 \\
\mathrm{a}\end{array}$ & $\begin{array}{l}1.87 \mathrm{a} \\
\mathrm{bc}\end{array}$ & $\begin{array}{l}3.24 \mathrm{a} \\
\mathrm{b}\end{array}$ & $3.33 \mathrm{ab}$ & $\begin{array}{l}3.4 \\
2 \mathrm{a}\end{array}$ & $\begin{array}{l}3.35 \\
\mathrm{ab}\end{array}$ & $\begin{array}{l}3.26 \\
\mathrm{a}\end{array}$ & $3.17 \mathrm{a}$ \\
\hline $\begin{array}{l}\mathrm{T}_{5^{-}} \text {Pseudomonas } \\
\text { fluorescence } 1 \%\end{array}$ & $\begin{array}{l}2.31 b \\
\text { cde }\end{array}$ & $\begin{array}{l}3.14 c \\
d e\end{array}$ & $\begin{array}{l}2.37 \\
\mathrm{~cd}\end{array}$ & $\begin{array}{l}2.03 \\
\mathrm{a}\end{array}$ & $1.56 \mathrm{~g}$ & $\begin{array}{l}3.16 c \\
\text { def }\end{array}$ & $3.26 \mathrm{f}$ & $\begin{array}{l}3.3 \\
3 \mathrm{~b}\end{array}$ & $\begin{array}{l}3.20 \\
\mathrm{e}\end{array}$ & $\begin{array}{l}3.14 \\
\mathrm{bc}\end{array}$ & $3.07 \mathrm{~b}$ \\
\hline $\begin{array}{l}\mathrm{T}_{6-} \text { Pseudomonas } \\
\text { fluorescence } 2 \%\end{array}$ & $\begin{array}{l}2.30 \mathrm{c} \\
\text { def }\end{array}$ & $\begin{array}{l}3.10 c \\
\text { de }\end{array}$ & $\begin{array}{l}2.36 \\
\mathrm{~cd}\end{array}$ & \begin{tabular}{|l}
2.08 \\
$\mathrm{a}$
\end{tabular} & $\begin{array}{l}1.68 \mathrm{e} \\
\mathrm{fg}\end{array}$ & $3.13 \mathrm{f}$ & $3.25 \mathrm{f}$ & $\begin{array}{l}3.3 \\
3 \mathrm{~b}\end{array}$ & $\begin{array}{l}3.20 \\
\mathrm{e}^{-3.0}\end{array}$ & $\begin{array}{l}3.15 \\
b c\end{array}$ & $3.07 \mathrm{~b}$ \\
\hline $\begin{array}{l}\mathrm{T}_{7^{-}} \text {Hot water } \\
\left(45^{0} \pm 2^{0} \mathrm{c}\right)\end{array}$ & $2.08 \mathrm{~g}$ & $\begin{array}{l}3.03 \mathrm{~d} \\
\mathrm{e}^{-1}\end{array}$ & $\begin{array}{l}2.36 \\
\mathrm{~cd}\end{array}$ & $\begin{array}{l}2.09 \\
\mathrm{a}\end{array}$ & $\begin{array}{l}1.74 \mathrm{c} \\
\mathrm{de}\end{array}$ & $\begin{array}{l}3.15 \mathrm{e} \\
\mathrm{f}\end{array}$ & $3.26 \mathrm{ef}$ & $\begin{array}{l}3.3 \\
3 \mathrm{~b}\end{array}$ & $3.2 \mathrm{e}$ & $\begin{array}{l}3.13 \\
\mathrm{bc}\end{array}$ & $3.05 \mathrm{c}$ \\
\hline $\begin{array}{l}\mathrm{T}_{8^{-}} \text {Hot water } \\
\left(35^{0} \pm 2^{0} \mathrm{c}\right)\end{array}$ & $\begin{array}{l}2.27 \mathrm{c} \\
\text { def }\end{array}$ & $\begin{array}{l}3.14 \mathrm{c} \\
\mathrm{d}\end{array}$ & $\begin{array}{l}2.31 \\
\mathrm{~d}\end{array}$ & $\begin{array}{l}2.12 \\
\mathrm{a}\end{array}$ & $\begin{array}{l}1.77 \mathrm{c} \\
\mathrm{de}\end{array}$ & $\begin{array}{l}3.15 \mathrm{~d} \\
\text { ef }\end{array}$ & $\begin{array}{l}3.27 \mathrm{de} \\
\mathrm{f}\end{array}$ & $\begin{array}{l}3.3 \\
3 b\end{array}$ & $\begin{array}{l}3.21 \\
\mathrm{e}\end{array}$ & $\begin{array}{l}3.16 \\
b\end{array}$ & $\begin{array}{l}3.04 \mathrm{~b} \\
\mathrm{~cd}\end{array}$ \\
\hline $\begin{array}{ll}\mathrm{T}_{9^{-}} & \text {Chitosan } \\
0.5 \% & \end{array}$ & $\begin{array}{l}2.09 f \\
\mathrm{~g}\end{array}$ & $\begin{array}{l}3.07 \mathrm{~d} \\
\mathrm{e}\end{array}$ & $\begin{array}{l}2.43 \\
\mathrm{~cd}\end{array}$ & $\begin{array}{l}2.08 \\
\mathrm{a}\end{array}$ & $\begin{array}{l}1.74 \mathrm{c} \\
\mathrm{de}\end{array}$ & $\begin{array}{l}3.20 \mathrm{~b} \\
\text { cde }\end{array}$ & $\begin{array}{l}3.30 \mathrm{ab} \\
\text { cde }\end{array}$ & $\begin{array}{l}3.3 \\
5 b\end{array}$ & $\begin{array}{l}3.27 \\
\mathrm{c}\end{array}$ & $\begin{array}{l}3.15 \\
\mathrm{bc}\end{array}$ & $\begin{array}{l}3.05 \mathrm{~b} \\
\mathrm{c}\end{array}$ \\
\hline $\mathrm{T}_{10^{-}}$Chitosan $1 \%$ & $\begin{array}{l}2.20 \mathrm{~d} \\
\text { efg }\end{array}$ & $3.00 \mathrm{e}$ & $\begin{array}{l}2.50 \\
\mathrm{c}\end{array}$ & $\begin{array}{l}2.09 \\
\mathrm{a}\end{array}$ & $\begin{array}{l}1.74 \mathrm{c} \\
\mathrm{de}\end{array}$ & $\begin{array}{l}3.22 \mathrm{a} \\
\mathrm{bc}\end{array}$ & $\begin{array}{l}3.28 \mathrm{de} \\
\text { f }\end{array}$ & $\begin{array}{l}3.3 \\
3 b\end{array}$ & $\begin{array}{l}3.26 \\
c d\end{array}$ & $\begin{array}{l}3.14 \\
b c\end{array}$ & $\begin{array}{l}3.05 b \\
c\end{array}$ \\
\hline $\begin{array}{ll}\mathrm{T}_{11^{-}} & \text {Guar } \\
\text { powder } 1 \% & \text { gum }\end{array}$ & $2.59 \mathrm{a}$ & $\begin{array}{l}3.34 \mathrm{a} \\
\mathrm{b}\end{array}$ & $\begin{array}{l}2.71 \\
\mathrm{a}\end{array}$ & $\begin{array}{l}2.26 \\
\mathrm{a}\end{array}$ & $\begin{array}{l}.83 b \\
\mathrm{~cd}\end{array}$ & $\begin{array}{l}3.23 \mathrm{a} \\
\mathrm{b}\end{array}$ & $\begin{array}{l}3.29 \mathrm{~cd} \\
\text { ef }\end{array}$ & $\begin{array}{l}3.3 \\
4 \mathrm{~b}\end{array}$ & $\begin{array}{l}3.27 \\
\mathrm{~cd}\end{array}$ & $\begin{array}{l}3.15 \\
b c\end{array}$ & $3.07 \mathrm{~b}$ \\
\hline $\begin{array}{ll}\mathrm{T}_{12^{-}} & \text {Guar } \\
\text { powder } 2 \% & \text { gum } \\
\end{array}$ & $\begin{array}{l}2.42 \mathrm{a} \\
\mathrm{bc}\end{array}$ & $\begin{array}{l}3.23 b \\
c\end{array}$ & $\begin{array}{l}2.50 \\
\mathrm{c}\end{array}$ & $\begin{array}{l}2.06 \\
\mathrm{a}\end{array}$ & $\begin{array}{l}1.81 \mathrm{~b} \\
\text { cde }\end{array}$ & $\begin{array}{l}3.20 b \\
\text { cde }\end{array}$ & $\begin{array}{l}3.32 \mathrm{ab} \\
\mathrm{cd}\end{array}$ & $\begin{array}{l}3.3 \\
6 \mathrm{~b} \\
\end{array}$ & $\begin{array}{l}3.27 \\
\mathrm{~cd}\end{array}$ & $\begin{array}{l}3.16 \\
\mathrm{~b}\end{array}$ & $3.07 \mathrm{~b}$ \\
\hline $\begin{array}{l}\mathrm{T}_{13^{-}} \text {Gum acacia } \\
10 \%\end{array}$ & $2.60 \mathrm{a}$ & $3.37 \mathrm{a}$ & $\begin{array}{l}2.73 \\
\mathrm{a}\end{array}$ & $\begin{array}{l}2.38 \\
\mathrm{a}\end{array}$ & $1.98 \mathrm{a}$ & $\begin{array}{l}3.26 \mathrm{a} \\
\mathrm{b}\end{array}$ & $3.34 \mathrm{a}$ & $\begin{array}{l}3.4 \\
1 \mathrm{a}\end{array}$ & $\begin{array}{l}3.35 \\
\mathrm{ab}\end{array}$ & $\begin{array}{l}3.28 \\
\mathrm{a}\end{array}$ & $3.19 \mathrm{a}$ \\
\hline $\begin{array}{l}\mathrm{T}_{14^{-}} \text {Gum acacia } \\
20 \%\end{array}$ & $\begin{array}{l}2.40 \mathrm{a} \\
\mathrm{bcd}\end{array}$ & $\begin{array}{l}3.12 c \\
\text { de }\end{array}$ & $\begin{array}{l}2.50 \\
\mathrm{c}\end{array}$ & $\begin{array}{l}2.00 \\
\mathrm{a}\end{array}$ & $\begin{array}{l}1.76 c \\
d e\end{array}$ & $3.27 \mathrm{a}$ & $\begin{array}{l}3.33 \mathrm{ab} \\
\mathrm{c}\end{array}$ & $\begin{array}{l}3.4 \\
3 \mathrm{a}\end{array}$ & $\begin{array}{l}3.36 \\
\mathrm{a}\end{array}$ & $\begin{array}{l}3.27 \\
\mathrm{a}\end{array}$ & $3.18 \mathrm{a}$ \\
\hline $\mathrm{T}$ & $\begin{array}{l}2.17 \mathrm{e} \\
\mathrm{fg}\end{array}$ & $\begin{array}{l}3.02 \mathrm{~d} \\
\mathrm{e}\end{array}$ & $\begin{array}{l}2.30 \\
\mathrm{~d}\end{array}$ & $\begin{array}{l}2.01 \\
\mathrm{a} \\
\end{array}$ & $\begin{array}{l}1.58 \mathrm{f} \\
\mathrm{g}\end{array}$ & $\begin{array}{l}3.21 \mathrm{a} \\
\mathrm{bcd}\end{array}$ & $\begin{array}{l}3.29 b c \\
\text { def }\end{array}$ & $\begin{array}{l}3.3 \\
3 \mathrm{~b} \\
\end{array}$ & $\begin{array}{l}3.25 \\
d\end{array}$ & $\begin{array}{l}3.13 \\
\mathrm{bc}\end{array}$ & $\begin{array}{l}3.02 \mathrm{c} \\
\mathrm{d}\end{array}$ \\
\hline $\begin{array}{l}\text { Least Significant } \\
\text { Difference } \\
(\mathbf{P} \leq \mathbf{0 . 0 5})\end{array}$ & 0.22 & 0.13 & 0.1 & 0.44 & 0.14 & 0.06 & 0.0 & $\begin{array}{l}\mathbf{0 . 0} \\
\mathbf{3}\end{array}$ & 0.02 & 0.04 & $\mathbf{0 . 0 3}$ \\
\hline
\end{tabular}

**Means with the same letter are not significantly different 
Table.7 Changes in ascorbic acid content (mg/100g of pulp)

\begin{tabular}{|c|c|c|c|c|c|c|c|c|c|c|c|}
\hline \multirow{3}{*}{ Treatments } & \multicolumn{11}{|c|}{ Days after treatments } \\
\hline & \multicolumn{5}{|c|}{ Rainy season } & \multicolumn{6}{|c|}{ Winter season } \\
\hline & 2 & 4 & 6 & 8 & 10 & 2 & 4 & 6 & 8 & 10 & 12 \\
\hline $\mathrm{T}_{1}-\mathrm{CaCl}_{2} 1 \%$ & $161.57 f$ & $154.21 \mathrm{~h}$ & $\begin{array}{l}149.41 \\
\text { ef }\end{array}$ & $\begin{array}{l}142.1 \\
6 \mathrm{e}\end{array}$ & $\begin{array}{l}135.24 \\
\text { gh }\end{array}$ & $\begin{array}{l}248.36 c \\
\text { def }\end{array}$ & $\begin{array}{l}243.2 \\
5 \mathrm{~cd}\end{array}$ & $\begin{array}{l}236.1 \\
5 \mathrm{e}\end{array}$ & $\begin{array}{l}230.4 \\
5 \mathrm{~g}\end{array}$ & $\begin{array}{l}225.3 \\
1 \mathrm{de}\end{array}$ & $\begin{array}{l}220.6 \\
1 \mathrm{de}\end{array}$ \\
\hline $\mathrm{T}_{2}-\mathrm{CaCl}_{2} 2 \%$ & $\begin{array}{l}55.17 \\
\text { le }\end{array}$ & $\begin{array}{l}159.61 d \\
e\end{array}$ & \begin{tabular}{|l|}
152.34 \\
cd
\end{tabular} & $\begin{array}{l}147.0 \\
0 \mathrm{c}\end{array}$ & $\begin{array}{l}140.27 \\
\text { de }\end{array}$ & $\begin{array}{l}253.16 a \\
b\end{array}$ & $\begin{array}{l}248.6 \\
1 \mathrm{a}\end{array}$ & $\begin{array}{l}242.0 \\
0 b\end{array}$ & $\begin{array}{l}236.1 \\
6 \mathrm{~cd}\end{array}$ & $\begin{array}{l}230.3 \\
4 c\end{array}$ & $\begin{array}{l}224.3 \\
9 \mathrm{c}\end{array}$ \\
\hline $\begin{array}{l}\mathrm{T}_{3^{-}} \text {Paraffin liquid } \\
5 \%\end{array}$ & $\begin{array}{l}167.24 \\
\mathrm{bc}\end{array}$ & $\begin{array}{l}162.00 \mathrm{~b} \\
\text { cde }\end{array}$ & \begin{tabular}{|l|}
157.18 \\
$\mathrm{~b}$ \\
\end{tabular} & $\begin{array}{l}151.0 \\
0 \mathrm{~b}\end{array}$ & $\begin{array}{l}145.32 \\
\mathrm{c}\end{array}$ & $\begin{array}{l}253.21 \mathrm{a} \\
\mathrm{b}\end{array}$ & $\begin{array}{l}249.3 \\
8 \mathrm{a} \\
\end{array}$ & $\begin{array}{l}245.3 \\
2 \mathrm{a}\end{array}$ & $\begin{array}{l}240.8 \\
4 a\end{array}$ & $\begin{array}{l}236.5 \\
1 \mathrm{a}\end{array}$ & $\begin{array}{l}230.3 \\
4 \mathrm{ab}\end{array}$ \\
\hline $\begin{array}{l}\mathrm{T}_{4}-\text { Paraffin liquid } \\
10 \%\end{array}$ & $\begin{array}{l}167.00 \\
\text { bc }\end{array}$ & $\begin{array}{l}163.14 a \\
b\end{array}$ & \begin{tabular}{|l|}
158.34 \\
$\mathrm{ab}$
\end{tabular} & $\begin{array}{l}152.2 \\
4 \mathrm{~b}\end{array}$ & $\begin{array}{l}146.23 \\
\mathrm{bc}\end{array}$ & $\begin{array}{l}253.17 \mathrm{a} \\
\mathrm{b}\end{array}$ & $\begin{array}{l}248.3 \\
1 \mathrm{a} \\
\end{array}$ & $\begin{array}{l}244.1 \\
6 \mathrm{a}\end{array}$ & $\begin{array}{l}239.6 \\
4 \mathrm{ab}\end{array}$ & $\begin{array}{l}235.6 \\
1 \mathrm{ab}\end{array}$ & $\begin{array}{l}229.1 \\
6 \mathrm{ab}\end{array}$ \\
\hline $\begin{array}{l}\mathrm{T}_{5^{-}} \text {Pseudomonas } \\
\text { fluorescence } 1 \%\end{array}$ & $\begin{array}{l}164.00 \\
\text { de }\end{array}$ & $\begin{array}{l}156.00 \mathrm{~g} \\
\mathrm{~h}\end{array}$ & \begin{tabular}{|l|}
150.05 \\
def
\end{tabular} & $\begin{array}{l}144.3 \\
1 \mathrm{de}\end{array}$ & $\begin{array}{l}137.26 \mathrm{f} \\
\mathrm{g}\end{array}$ & $\begin{array}{l}247.00 \mathrm{e} \\
\mathrm{f}\end{array}$ & $\begin{array}{l}241.2 \\
8 \mathrm{~cd} \\
\end{array}$ & $\begin{array}{l}235.3 \\
4 \mathrm{ef}\end{array}$ & $\begin{array}{l}230.6 \\
1 \mathrm{fg}\end{array}$ & $\begin{array}{l}225.6 \\
4 \mathrm{de} \\
\end{array}$ & $\begin{array}{l}219.6 \\
1 \mathrm{de}\end{array}$ \\
\hline $\begin{array}{l}\mathrm{T}_{6-} \text { Pseudomonas } \\
\text { fluorescence } 2 \%\end{array}$ & $\begin{array}{l}163.42 \\
\text { ef }\end{array}$ & $\begin{array}{l}156.65 f \\
\mathrm{gh}\end{array}$ & \begin{tabular}{|l|}
150.82 \\
cde \\
\end{tabular} & $\begin{array}{l}142.2 \\
5 \mathrm{e} \\
\end{array}$ & $\begin{array}{l}135.21 \\
\text { gh }\end{array}$ & $\begin{array}{l}248.21 \mathrm{c} \\
\text { def }\end{array}$ & $\begin{array}{l}243.3 \\
4 \mathrm{bc} \\
\end{array}$ & $\begin{array}{l}237.5 \\
7 \mathrm{~d} \\
\end{array}$ & $\begin{array}{l}231.0 \\
4 \mathrm{fg}\end{array}$ & $\begin{array}{l}226.5 \\
4 d\end{array}$ & $\begin{array}{l}220.3 \\
1 \mathrm{de}\end{array}$ \\
\hline $\begin{array}{l}\mathrm{T}_{7^{-}} \quad \text { Hot } \quad \text { water } \\
\left(45^{0} \pm 2^{0} \mathrm{c}\right)\end{array}$ & $\begin{array}{l}163.12 \\
\text { ef }\end{array}$ & $155.26 \mathrm{~h}$ & $148.21 \mathrm{f}$ & $\begin{array}{l}144.2 \\
8 \mathrm{de}\end{array}$ & $\begin{array}{l}138.00 \\
\text { ef }\end{array}$ & $245.62 f$ & $\begin{array}{l}239.2 \\
7 \mathrm{de} \\
\end{array}$ & $\begin{array}{l}233.6 \\
4 f\end{array}$ & $\begin{array}{l}227.3 \\
4 \mathrm{~h} \\
\end{array}$ & $\begin{array}{l}222.6 \\
1 \mathrm{f}\end{array}$ & $\begin{array}{l}218.1 \\
6 \mathrm{ef}\end{array}$ \\
\hline $\begin{array}{l}\mathrm{T}_{8^{-}} \quad \text { Hot water } \\
\left(35^{0} \pm 2^{0} \mathrm{c}\right)\end{array}$ & $\begin{array}{l}166.00 \\
\text { bcd }\end{array}$ & $\begin{array}{l}158.36 \mathrm{e} \\
\mathrm{fg}\end{array}$ & \begin{tabular}{|l|}
$\begin{array}{l}151.45 \\
\text { cde }\end{array}$ \\
\end{tabular} & $\begin{array}{l}144.3 \\
4 \mathrm{de}\end{array}$ & $\begin{array}{l}135.42 \\
\mathrm{gh}\end{array}$ & $\begin{array}{l}251.00 \mathrm{a} \\
\mathrm{bc}\end{array}$ & $\begin{array}{l}245.3 \\
1 \mathrm{~b} \\
\end{array}$ & $\begin{array}{l}240.2 \\
1 \mathrm{bc}\end{array}$ & $\begin{array}{l}234.5 \\
4 \mathrm{de}\end{array}$ & $\begin{array}{l}229.6 \\
1 \mathrm{c} \\
\end{array}$ & $\begin{array}{l}221.5 \\
2 \mathrm{~cd}\end{array}$ \\
\hline $\mathrm{T}_{9^{-}}$Chito & $\begin{array}{l}165.16 \\
\text { cde }\end{array}$ & $\begin{array}{l}158.32 \mathrm{e} \\
\mathrm{fg}\end{array}$ & \begin{tabular}{|l|}
152.37 \\
cde
\end{tabular} & $\begin{array}{l}146.2 \\
9 \mathrm{~cd}\end{array}$ & $\begin{array}{l}140.28 \\
\text { de }\end{array}$ & $\begin{array}{l}247.00 \mathrm{e} \\
\mathrm{f}\end{array}$ & $\begin{array}{l}241.3 \\
1 \mathrm{~cd} \\
\end{array}$ & $\begin{array}{l}235.0 \\
\text { Oef }\end{array}$ & $\begin{array}{l}230.6 \\
1 \mathrm{fg}\end{array}$ & $\begin{array}{l}224.3 \\
5 \mathrm{ef}\end{array}$ & $\begin{array}{l}219.6 \\
1 \mathrm{de}\end{array}$ \\
\hline $\mathrm{T}_{10^{-}}$Chitosan $1 \%$ & $\begin{array}{l}165.32 \\
\text { cde }\end{array}$ & $\begin{array}{l}159.45 \mathrm{e} \\
\mathrm{f}\end{array}$ & \begin{tabular}{|l|}
153.12 \\
$\mathrm{c}$
\end{tabular} & $\begin{array}{l}147.2 \\
6 \mathrm{c}\end{array}$ & $\begin{array}{l}141.33 \\
\mathrm{~d}\end{array}$ & $\begin{array}{l}250.31 \mathrm{a} \\
\text { bcd }\end{array}$ & $\begin{array}{l}242.3 \\
0 \mathrm{c} \\
\end{array}$ & $\begin{array}{l}236.3 \\
1 \mathrm{de}\end{array}$ & $\begin{array}{l}230.0 \\
0 \mathrm{~g}\end{array}$ & $\begin{array}{l}223.0 \\
\text { Of }\end{array}$ & $\begin{array}{l}215.6 \\
1 f\end{array}$ \\
\hline $\mathrm{T}_{11^{-}}$Gua & $\begin{array}{l}168.05 \\
\mathrm{ab}\end{array}$ & $\begin{array}{l}162.31 b \\
\mathrm{~cd}\end{array}$ & \begin{tabular}{|l|}
157.61 \\
$\mathrm{~b}$ \\
\end{tabular} & $\begin{array}{l}151.3 \\
4 \mathrm{~b}\end{array}$ & $\begin{array}{l}148.36 \\
\mathrm{ab}\end{array}$ & $\begin{array}{l}249.31 \mathrm{c} \\
\mathrm{de}\end{array}$ & $\begin{array}{l}245.3 \\
1 \mathrm{~b} \\
\end{array}$ & $\begin{array}{l}239.1 \\
3 \mathrm{c} \\
\end{array}$ & $\begin{array}{l}232.6 \\
5 \mathrm{ef}\end{array}$ & $\begin{array}{l}226.3 \\
5 \mathrm{~d} \\
\end{array}$ & $\begin{array}{l}222.3 \\
1 \mathrm{~cd} \\
\end{array}$ \\
\hline $\mathrm{T}_{12-}$ - Guar gum $2 \%$ & $\begin{array}{l}167.27 \\
\text { bc }\end{array}$ & $\begin{array}{l}163.19 a \\
b\end{array}$ & $\begin{array}{l}158.27 \\
\mathrm{ab}\end{array}$ & $\begin{array}{l}152.3 \\
4 \mathrm{~b}\end{array}$ & $\begin{array}{l}145.23 \\
\mathrm{c}\end{array}$ & $\begin{array}{l}247.61 d \\
\text { ef }\end{array}$ & $\begin{array}{l}243.2 \\
1 b c\end{array}$ & $\begin{array}{l}237.6 \\
1 d\end{array}$ & $\begin{array}{l}231.2 \\
6 f g\end{array}$ & $\begin{array}{l}225.1 \\
3 \mathrm{de}\end{array}$ & $\begin{array}{l}220.6 \\
1 \mathrm{de}\end{array}$ \\
\hline $\begin{array}{l}\mathrm{T}_{13^{-}} \text {Gum acacia } \\
10 \%\end{array}$ & $\begin{array}{l}170.00 \\
\mathrm{a}\end{array}$ & $165.31 \mathrm{a}$ & \begin{tabular}{|l|}
160.20 \\
$\mathrm{a}$ \\
\end{tabular} & $\begin{array}{l}155.1 \\
5 \mathrm{a} \\
\end{array}$ & $\begin{array}{l}150.10 \\
\mathrm{a}\end{array}$ & $\begin{array}{l}250.15 b \\
\text { cde }\end{array}$ & $\begin{array}{l}245.1 \\
6 \mathrm{~b} \\
\end{array}$ & $\begin{array}{l}241.3 \\
1 \mathrm{~b}\end{array}$ & $\begin{array}{l}237.6 \\
1 \mathrm{bc}\end{array}$ & $\begin{array}{l}234.6 \\
1 \mathrm{~b}\end{array}$ & $\begin{array}{l}229.0 \\
0 \mathrm{~b}\end{array}$ \\
\hline $\begin{array}{l}\mathrm{T}_{14^{-}} \text {Gum acacia } \\
20 \%\end{array}$ & $\begin{array}{l}168.05 \\
\mathrm{ab}\end{array}$ & $\begin{array}{l}163.00 \mathrm{a} \\
\mathrm{bc}\end{array}$ & \begin{tabular}{|l|}
158.34 \\
$\mathrm{ab}$
\end{tabular} & $\begin{array}{l}152.0 \\
0 \mathrm{~b}\end{array}$ & $\begin{array}{l}148.10 \\
a b\end{array}$ & $253.42 \mathrm{a}$ & $\begin{array}{l}249.6 \\
1 \mathrm{a} \\
\end{array}$ & $\begin{array}{l}244.3 \\
1 \mathrm{a}\end{array}$ & $\begin{array}{l}240.3 \\
1 \mathrm{a}\end{array}$ & $\begin{array}{l}236.1 \\
0 \mathrm{ab}\end{array}$ & $\begin{array}{l}232.1 \\
5 \mathrm{a}\end{array}$ \\
\hline $\mathrm{T}_{15^{-}}$Control & $\begin{array}{l}167.19 \\
\mathrm{bc}\end{array}$ & $\begin{array}{l}160.18 \mathrm{c} \\
\mathrm{de}\end{array}$ & \begin{tabular}{|l|}
152.61 \\
$\mathrm{c}$ \\
\end{tabular} & $\begin{array}{l}145.2 \\
6 \mathrm{~cd} \\
\end{array}$ & $\begin{array}{l}133.28 \\
\mathrm{~h}\end{array}$ & $246.00 f$ & $\begin{array}{l}238.0 \\
9 \mathrm{e} \\
\end{array}$ & $\begin{array}{l}230.6 \\
1 \mathrm{~g} \\
\end{array}$ & $\begin{array}{l}223.0 \\
6 \mathrm{i} \\
\end{array}$ & $\begin{array}{l}218.0 \\
0 \mathrm{~g}\end{array}$ & $\begin{array}{l}210.3 \\
1 \mathrm{~g}\end{array}$ \\
\hline $\begin{array}{l}\text { Least Significant } \\
\text { Difference } \\
(\mathrm{P} \leq 0.05)\end{array}$ & 2.39 & 2.87 & 2.34 & 2.37 & 2.37 & 3.17 & 2.33 & 1.42 & 2.16 & 1.73 & 3.13 \\
\hline
\end{tabular}

**Means with the same letter are not significantly different

In rainy season, on 10 days after treatment, TSS was found maximum $\left(5.95^{\mathrm{O}}\right.$ brix $)$ with gum acacia $10 \%$, followed by guar gum $1 \%$ $\left(5.93^{\circ}\right.$ brix $)$ and statistically at par with paraffin liquid $5 \%, 10 \%$, and gum acacia $20 \%$, whereas, it was minimum $\left(5.16^{\circ}\right.$ brix $)$ under control. Similarly, in winter season, on 12 days after treatment, it was found maximum $\left(11.26^{\mathrm{O}}\right.$ brix $)$ with gum acacia
$10 \%$ which was statistically at par with the gum acacia $20 \%$, paraffin liquid $5 \%$ and $10 \%$. The increase in TSS and sugar content during storage may possibly be due to hydrolysis of starch into sugars as on complete hydrolysis of starch no further increase occurs and subsequently a decline in these parameters is predictable as they along with other organic acids are primary substrate for respiration 
(Wills et al., 1980). Kagzi lime fruits treated with coconut oil recorded minimum $(8.4 \%)$ increase in TSS followed by (8.5\%) liquid paraffin wax (Bisen et al., 2012).

\section{Total sugar (TS)}

In rainy season, on 10 days after treatment, total sugar (Table 5) content was found maximum (3.93) with guar gum $1 \%$ and gum acacia $10 \%$ and statistically at par with paraffin liquid 5\% (3.89), gum acacia 20\% (3.89) and paraffin liquid $10 \%$ (3.69), whereas, it was minimum(3.13) under control. However, in winter season, on 12 days after treatment, total sugar content was found maximum (6.66) with gum acacia $20 \%$ and statistically at par with gum acacia $10 \%$ (6.64), paraffin liquid 10\% (6.62) and paraffin liquid 5\% (6.60), whereas, it was minimum (6.04) with calcium chloride $1 \%$.

\section{Reducing sugar}

In rainy season, on 10 days after treatment, reducing sugar content (Table 6) was found maximum (1.98) with gum acasia $10 \%$ and statistically at par with paraffin liquid 5\% $(1.96 \%)$ paraffin liquid $10 \%$ (1.87). In winter season similarly, maximum (3.19)reducing sugar content was recorded with gum acasia $10 \%$ and it was statistically at par with paraffin liquid 5\% (3.18), paraffin liquid 10\% (3.17) and gum acasia 20\%(3.18).

\section{Ascorbic acid}

The ascorbic acid content (Table 7) was decreased in all the treatments as the storage period advanced. In rainy season, on 10 days after treatment, the ascorbic acid content was found highest $(150.1 \mathrm{mg} / 100 \mathrm{~g}$ of pulp) with gum acacia $10 \%$ and statistically at par with the guar gum $1 \%$ and gum acacia $20 \%$, whereas, it was lowest $(133.28 \mathrm{mg} / 100 \mathrm{~g}$ of pulp) under control. However, in winter season, on 12 days after treatment, it was found maximum (232.15mg/100g of pulp) with gum acacia $20 \%$ and statistically at par with the paraffin liquid $5 \%(230.34 \mathrm{mg} / 100 \mathrm{~g}$ of pulp) and $10 \%(229.16 \mathrm{mg} / 100 \mathrm{~g}$ of pulp), whereas, it was minimum $(210.31 \mathrm{mg} / 100 \mathrm{~g}$ of pulp) under control. The ascorbic acid content (5.81 mg/100g) was more in peach fruits treated with paraffin liquid (20\%) as compared to fruits under control (2.81 mg/100g) (Elham and Sawsan, 2013).

In conclusion, the fruit retention percentage in both the rainy and winter season was found maximum with paraffin liquid $10 \%$. Physiological loss in weight was minimum in fruits treated with the guar gum $1 \%$ in rainy season and paraffin 5\% and gum acacia $10 \%$ in winter season. Disease incidence was found minimum in the fruits treated with paraffin liquid $5 \%$ in both the seasons. Regarding other horticultural traits like TSS, ascorbic acid, total sugar, reducing sugar, content were recorded maximum with gum acacia $10 \%$ in rainy season guava fruits and gum acacia10\% and $20 \%$ in winter season, respectively. From this experiment, it is concluded that winter season guava fruits could be store well as compared to rainy season fruits. Edible coating materials showed the better results in terms of extending the shelf life as well as the qualities of guava fruits than the other chemicals in both rainy and winter season.

\section{References}

Akamine, E.K., Goo, T. 1979. Respiration and ethylene production in fruits of species and cultivars of Psydium and species of Eugenia. J. Am. Soc. Hort. Sci., 104: 632-635.

AOAC. 1984. Official methods of analysis $\left(14^{\text {th }} \mathrm{ed}\right)$. Washington, DC: Association of Official Agriculture Chemist

Ayranci, E., S. Tunc. 2003. A method for the measurement of the oxygen permeability and the development of edible films to reduce the rate of 
oxidative reactions in fresh foods. Food Chem., 80: 423-431.

Baldwin, E.A., Burns, J.K., Kazokas, W., Brecht, .J.K, Hagenmaier, R.D., Bender, R., Pesise. 1999. Effect of two edible coatings with different permeability characteristics on mango (MangiferaindicaL.) ripening during storage. Posthar Biol. Technol., 17: 215-226.

Bisen, A., Pandey, S.K., Patel, N. 2012. Effect of skin coatings on prolonging shelf life of kagzi lime fruits (Citrus aurantifolia Swingle. J. Food Sci. Technol., 49(6): 753-759.

Bose, T.K., Mitra, S.K 2001. Guava.In: Fruits: Tropical and subtropical. NayaPrakasan, Kolkata. pp. 280-303.

Elham, Z., Abd El-Motty, Sawsan, Y., ElFaham. 2013. Effect of oil coating and different wrapping materials on prolonging storage periods of Florida Prince Peach fruits. J. Appl. Sci. Res., 9(4): 2927-2937.

Garg, R.C., Ram, H.B. 1973. A note on the effect of wax emulsion treatment on mango cv. Lucknow Safeda. Prog. Hort., 5: 35-39.

Gomez, K.A., Gomez, A.A. 1983. Problem data. In: Statistical procedures for Agricultural Research (2 ${ }^{\text {nd }}$ edition), Wiley-lnter science publication (John Wiley and Sons); New York, USA, pp 275-315.
Mazumdar, B.C., Majumder, K. 2003. Determination of chemical constituents. In: Methods on physico-chemical Analysis of Fruits. Delhi: Daya Publishing House. New Delhi, India

Mercado-Silva, E., Benito-Bautista, P., Garcia-Velasco, M.A. 1998. Fruit development, harvest index and ripening changes of guavas produced in central Mexico. Posthar Bio and Tech., 13: $143-150$.

Pal, P.K., Ahmad, M.S., Roy, S.K., Singh, M. 2004. Influence of storage environment, surface coating, and individual shrink wrapping on quality assurance of guava (Psidium guajava) fruits. Plant Foods Hum. Nutr., 59: 67-72.

Passam, H.C. 1982. Storage of some local and introduced mango cultivars grown in Trinidad. Scientia Hort., 16: 171-177.

Rangana, S. 1977. In: Manual of analysis of fruits and vegetable products. Tata and Mc. Graw Hill Publishing company limited: New Delhi, India

Wang, H., Huang, C.C., Chienh, S. 1964. A study on the storage and post-harvest treatment of mango fruits. Rep. Fengshan. Trop. Hort. Exp. Stat., Taiwan, 32: 7.

Wills, R.B.H., Bembridge, P.A., Scott, K.J. 1980. Use of flesh firmness and other objective tests to determine consumer acceptability of Delicious apples. Aust. J. Exp. Agri. and Ainm., 20: 252-256.

\section{How to cite this article:}

Piyali Dutta, Nilesh Bhowmick, Surajit Khalko, Arunava Ghosh and Swapan Kr. Ghosh. 2017. Postharvest Treatments on Storage Life of Guava (Psidium guajava L.) in Himalayan Terai Region of West Bengal, India. Int.J.Curr.Microbiol.App.Sci. 6(3): 1831-1842. doi: https://doi.org/10.20546/ijcmas.2017.603.209 